\title{
Evidence for Gaze Feedback to the Cat Superior Colliculus: Discharges Reflect Gaze Trajectory Perturbations
}

\author{
Satoshi Matsuo, André Bergeron, and Daniel Guitton \\ Montreal Neurological Institute, McGill University, Montreal, Quebec, Canada, H3A2B4
}

\begin{abstract}
Rapid coordinated eye-head movements, called saccadic gaze shifts, displace the line of sight from one location to another. A critical structure in the gaze control circuitry is the superior colliculus (SC) of the midbrain, which drives gaze saccades by relaying cortical commands to brainstem eye and head motor circuits. We proposed that the SC lies within a gaze feedback loop and generates an error signal specifying gaze position error (GPE), the distance between target and current gaze positions. We investigated this feedback hypothesis in cats by briefly stopping head motion during large $\left(\approx 50^{\circ}\right)$ gaze saccades made in the dark. This maneuver interrupted intended gaze saccades and briefly immobilized gaze (a plateau). After brake release, a corrective gaze saccade brought the gaze on goal. In the caudal SC, the firing frequency of a cell gradually increased to a maximum that just preceded the optimal gaze saccade encoded by the position of the cell and then declined back to zero near gaze saccade end. In brake trials, the activity level just preceding a brakeinduced plateau continued steadily during the plateau and waned to zero only near the end of the corrective saccade. The duration of neural activity was stretched to reflect the increased time to target acquisition, and firing frequency during a plateau was proportional to the GPE of the plateau. In comparison, in the rostral SC, the duration of saccade-related pauses in fixation cell activity increased as plateau duration increased. The data show that the cat's SC lies in a gaze feedback loop and that it encodes GPE.
\end{abstract}

Key words: superior colliculus; single-unit recording; saccades; gaze; eye-head coordination; feedback control; moving-hill hypothesis

\section{Introduction}

A dominant question in oculomotor control is how the spatially encoded signal on the motor map of the superior colliculus (SC) is transformed into the temporally encoded signal required by reticular gaze control circuits that receive collicular commands (for review, see Moschovakis et al., 1996; Scudder et al., 2002). It has been proposed that ocular saccades are controlled by a local brainstem feedback loop that compares desired with actual eye rotation to generate an eye motor-error signal that in turn drives the saccade burst generator until the error is nullified. In one hypothesis, the SC is within the feedback loop (for review, see Scudder et al., 2002; Guitton et al., 2003a,b): in primate, its output has been assumed to encode eye motor error either exclusively at the initially active site on the motor map (Waitzman et al., 1991) or by a spreading front of activity across the map that stops saccades when it invades the fixation zone (Munoz et al., 1991a,b; Munoz and Wurtz, 1995a,b).

If the SC is in a feedback loop, collicular discharges should respond to perturbations of saccadic eye movement trajectories. This important test has been attempted in the head-fixed monkey. Saccade trajectories were perturbed by using either electrophysiological or pharmacological approaches (Keller and Edel-

Received June 18, 2003; revised Jan. 21, 2004; accepted Jan. 22, 2004.

This work was supported by the Canadian Institutes of Health Research (CIHR). S.M. was supported by a grantin-aid for scientific research from the Japanese Ministry of Education, Science and Culture. A.B. was funded by a CIHR studentship. We thank W. Y. Choi for a very helpful critique.

Correspondence should be addressed to Dr. Daniel Guitton, Montreal Neurological Institute, 3801 University Street, Montreal, QC, Canada, H3A 2B4. E-mail: daniel.guitton@mcgill.ca.

DOI:10.1523/JNEUROSCI.5120-03.2004

Copyright $\odot 2004$ Society for Neuroscience $\quad 0270-6474 / 04 / 242760-14 \$ 15.00 / 0$ man, 1994; Munoz et al., 1996; Keller et al., 2000; Soetedjo et al., 2002a) as well as with blinks (Goossens and Van Opstal, 2000a,b). In these studies, monkeys compensated accurately for the perturbation, and gaze arrived on target. Concurrently, the firingfrequency profile of SC saccade-related neurons changed relative to that in unperturbed saccades. Specifically, discharge duration increased in relation to the increased overall duration of the eye movement necessary for the eye to get on target. The interpretation of the results varied greatly among the studies, varying from a support of the SC being in a feedback loop encoding either motor error (Munoz et al., 1996) or saccade end (Soetedjo et al., 2002a) or an unknown mechanism (Keller et al., 2000) to the argument that the SC functions open loop and sends to the pons a fixed number of spikes (Goossens and Van Opstal, 2000a,b).

Head-unrestrained gaze saccades are also thought to be controlled by a feedback loop that produces a signal encoding gaze position error (GPE), the distance between target and current gaze positions (for review, see Scudder et al., 2002; Guitton et al., 2003a,b). We proposed that cat SC efferents, tectoreticular neurons, encode GPE (Munoz et al., 1991a,b; Bergeron and Guitton, 2000, 2002; Bergeron et al., 2003). According to our moving-hill model, during a gaze shift, the locus of neural activity on the motor map continuously encodes GPE by moving rostrally from an initial location that encodes the overall direction and amplitude of the gaze shift to a final zero position in the fixation zone of the SC.

Gaze shifts are ideally suited for testing feedback to the SC, because they can be easily perturbed by briefly braking the head trajectory in-flight. Head perturbations are a natural phenomenon that occurs readily in the daily life of animals and in human 
sporting activities. In comparison, it is unnatural to perturb head-fixed saccades by deactivating or stimulating brainstem circuits. Our recordings in the SC during mechanical interference with gaze motion support the hypothesis that the cat SC is in a gaze feedback loop, and that it encodes GPE.

\section{Materials and Methods}

Animal preparation. All of the surgical and experimental protocols were approved by the Animal Care Committee of the Montreal Neurological Institute and complied with the Canadian Council on Animal Care policy on the use of laboratory animals. Several cats underwent initial training and were chosen for their capacity to orient repeatedly and accurately to the target in our barrier paradigm (see Behavioral procedures). The data described here were obtained in four of the eight cats that provided the material for our previous papers describing the discharges of cat collicular cells during multiple-step gaze shifts (Bergeron and Guitton, 2000; Bergeron et al., 2003). The four cats (K, N, S, and W) were prepared for chronic single-unit recording in the SC. Anesthesia was induced with an intramuscular injection of ketamine hydrochloride $(10 \mathrm{mg} / \mathrm{kg})$. The cats were then intubated and maintained on anesthesia using halothane. During surgery, heart rate, respiratory rate, and body temperature were monitored. A wire coil consisting of three turns of Teflon-coated multistrand stainless-steel wire (California Fine Wire, Grover Beach, CA) was sutured to the sclera of one eye for recording eye movements (Robinson, 1963). The wire leads passed subcutaneously to an acrylic skull explant that was anchored to the skull with T-shaped stainless-steel bolts. To permit access to the SC for single-unit recording, a stainless-steel cylinder, constructed to hold a small micropositioner (Narishige, Tokyo, Japan), was positioned vertically on the midline of the cranium surface at anteroposterior stereotaxic coordinate 0 (Berman, 1968).

To determine by antidromic stimulation whether the collicular cell being recorded was a tectoreticular neuron, we implanted into the predorsal bundle of cat $\mathrm{W}$ a bipolar concentric stimulating electrode (SNEX100; Kopf, Tujunga, CA), which was fixed to the explant. This electrode was lowered into the brainstem at an angle of $20-30^{\circ}$ posterior to the frontal plane, to a site within the predorsal bundle just rostral to the abducens nucleus (stereotaxic coordinates, posterior, 5.5; horizontal, -5.0; mediolaterial, 0) (Berman, 1968). The final stimulating-electrode position was determined by trial and error: while continuously stimulating, the electrode position was adjusted such that a recording microelectrode in the SC could record antidromically activated SC cells and/or strong evoked potentials.

The connectors for the eye coil, the recording cylinder, and the stimulating electrode were embedded in the explant. A screw, attached to the explant, held a second search coil used to measure head movements. A thin stainless-steel U-shaped crown was also embedded in the posterior perimeter of the explant for the purpose of attaching the cat's head to a universal joint, itself attached to a freely rotating vertical shaft (see Behavioral procedures).

After the surgery, an intramuscular injection of gentamicin $(15 \mathrm{mg} / \mathrm{kg})$ or cefazolin $(35 \mathrm{mg} / \mathrm{kg})$ was administered as a prophylactic measure against infection. This treatment was continued on a daily basis for 10 postoperative days. At the end of the surgery, an analgesic medication (buprenorphine hydrochloride; $0.01 \mathrm{mg} / \mathrm{kg}$ ) was given and continued for $2 \mathrm{~d}$. Cats recovered for at least $10 \mathrm{~d}$ before experimental procedures commenced.

Behavioral procedures. During the experiments, the alert cat was enveloped in a loosely fitting cloth bag and placed in an open-top box that gently restrained its body and limb movements. The animal box allowed full ranges in horizontal and vertical-up head motion except for downward head motion, which was restricted to $\sim 35^{\circ}$ below normal head posture. The U-shaped crown in the explant was attached, via two universal joints, to a vertical shaft rotating in low-friction bearings. The double universal joints permitted about $\pm 30^{\circ}$ of vertical head motion and minimized constraint on the animal's orienting behavior in the earth-horizontal plane, which is what we focused on here. Our previous studies have shown that experimental results are not affected by whether or not the animal's head is attached to the shaft-universal joint assembly
(Bergeron and Guitton, 2000, 2002). To perturb a horizontal gaze shift in-flight, the rotating shaft was unexpectedly halted by a friction clutch, attached to the shaft, which was activated at different times relative to the onset of head motion (determined by a velocity threshold).

To obtain memory-guided gaze shifts, we used a barrier paradigm (Munoz and Guitton, 1991). A cat faced an opaque barrier of variable width $\left(40-60^{\circ}\right)$ directly in front at a distance of $\sim 35 \mathrm{~cm}$. Initially, in a trial, there was no target of significance to the cat. A small food target on a holder was hidden behind the barrier and suddenly appeared at the edge of one of the two vertical sides. In this condition, the hungry cat naturally redirected its visual axis to the target and was fed. To obtain the time of target appearance, the rapidly moving food target intercepted infrared detectors located on the edges of the barrier, which generated a markerpulse fed to the computer. The horizontal trajectory of the target also was measured using a search coil attached to the food holder. This coil was sensitive to the translational motion of the target, because it moved far from the center of the field coil assembly in a path where the horizontal magnetic field was not uniform. The calibration of this coil signal was obtained by referring it to the actual physically measured target position. Using these two methods, our estimate of time of target appearance was within \pm 3 msec.

The barrier paradigm permitted a wide range of gaze shift amplitudes not only because of the variable barrier width, but also because the overall gaze shift required to fixate the target depended on the initial gaze position, which varied from trial to trial. Ambient lighting was provided by a fluorescent light with very fast decay time $(\sim 100 \mu \mathrm{sec})$. To obtain gaze shifts in complete darkness, to the remembered location of the target, ambient lighting was extinguished for $1 \mathrm{sec}$ beginning $120 \mathrm{msec}$ after an infrared detector was triggered by the passing food target. Gaze shifts in the light were obtained by keeping ambient lighting on. The occurrence of predictive movements was minimized by regularly changing the size of the barrier and the location where the target appeared.

We recorded cell activity in four randomly interposed trial types: the cat oriented in either the light or dark and with or without a head brake. In 20 and $80 \%$ of the trials, the cat oriented in the light and dark, respectively. The head brake was applied randomly and occurred in $50 \%$ of dark and light trials for durations ranging from 50 to $300 \mathrm{msec}$.

Recording procedures. We first explored the motor map of the SC using electrical stimulation (train duration, $300 \mathrm{msec}$; pulse width, $300 \mu \mathrm{m}$; frequency, $300 \mathrm{~Hz}$; current, 5-30 $\mu \mathrm{A}$ ) (Paré et al., 1994). The organization of the map was deduced on the basis of the amplitude and direction of gaze shifts evoked at different electrode positions (Paré et al., 1994). With this method, it was possible to predict the stereotaxic location of both the fixation zone in the rostral SC, and the locus of points that encoded horizontal gaze shifts. We then confirmed these locations by applying electrical stimulation and verifying that (1) for the fixation zone, gaze shifts could be interrupted, and (2) for the motor map, stimulation at different points along the predicted horizontal meridian evoked horizontal gaze shifts.

We recorded in the intermediate and deep layers, at $\sim 1.2-3 \mathrm{~mm}$ below the dorsal surface of the SC. The neurons we describe here presented the pattern of firing frequency previously described in our previous studies of identified tectoreticular neurons (Munoz and Guitton, 1991; Munoz et al., 1991a,b; Bergeron and Guitton, 2000, 2002; Bergeron et al., 2003). As we shall see in Results, for gaze shifts in the dark of optimal amplitude, this was the following: (1) a low-frequency prelude of activity that began $\sim 100$ msec after target appearance and gradually built up until the onset of the gaze shift, (2) a subsequent decline of firing frequency to zero at about the end of the gaze shift, (3) an open-ended movement field, meaning that the cell discharged for all of the movements larger than its optimal, and (4) for large gaze shifts, a peak discharge in cells of the rostral one-half of the SC that lagged gaze shift onset. Some of these criteria are in accordance with other descriptions of cat tectoreticular neurons projecting from the collicular motor map (Grantyn and Berthoz, 1985; Olivier et al., 1993). In addition, we verified in cat W whether the recorded cells were tectoreticular neurons by stimulating antidromically the main descending axons from the SC, at a site in the predorsal bundle just rostral to the abducens nucleus (see Animal preparation). Stimulation consisted of a biphasic current pulse $(30 \mu \mathrm{A})$ that 
Table 1. Cells described in this paper

\begin{tabular}{|c|c|c|c|c|c|c|}
\hline \multirow[b]{2}{*}{ Cells } & \multirow{2}{*}{$\begin{array}{l}\text { SC } \\
\text { location } \\
\text { (in } \mathrm{mm} \text { ) }\end{array}$} & \multicolumn{3}{|c|}{ Optimal GPE } & \multirow{2}{*}{$\begin{array}{l}\text { Number } \\
\text { trials } \\
\text { control }\end{array}$} & \multirow{2}{*}{$\begin{array}{l}\text { Number } \\
\text { trials } \\
\text { brake }\end{array}$} \\
\hline & & $\mathrm{GPE}_{\text {Ostim }}$ & $\mathrm{GPE}_{0 \mathrm{msgs}}$ & $\mathrm{GPE}_{\text {Ocontrol }}$ & & \\
\hline W51* & 0 & $\mathrm{Gl}$ & NC & 0 & 19 & 27 \\
\hline N38 & 0 & $\mathrm{Gl}$ & 0 & 0 & 5 & 6 \\
\hline $\mathrm{N} 40$ & 0 & $\mathrm{Gl}$ & 0 & 0 & 15 & 16 \\
\hline N42a & 0 & $\mathrm{Gl}$ & 0 & 0 & 13 & 19 \\
\hline W30 & 0 & $\mathrm{Gl}$ & NC & 0 & 6 & 4 \\
\hline К6а & 1.90 & 10 & NC & 11 & 23 & 8 \\
\hline K8 & 2.15 & 13 & NC & 18 & 25 & 23 \\
\hline W113c & 2.29 & 15 & NC & NC & 0 & 8 \\
\hline K10 & 2.56 & 10 & 19 & 12 & 22 & 21 \\
\hline W112d & 2.56 & 14 & 19 & 9 & 8 & 16 \\
\hline S45a & 2.74 & 19 & 22 & NC & 6 & 8 \\
\hline W110* & 2.79 & 18 & 23 & 16 & 21 & 21 \\
\hline K7 & 2.85 & 15 & 24 & 20 & 28 & 35 \\
\hline K1a & 3.06 & 28 & NC & 20 & 8 & 4 \\
\hline K10a & 3.06 & 28 & NC & NC & 12 & 20 \\
\hline K1 & 3.47 & 32 & 37 & 26 & 18 & 5 \\
\hline W113a* & 3.47 & 32 & 37 & 38 & 14 & 21 \\
\hline W113b* & 3.47 & 32 & 37 & 37 & 6 & 11 \\
\hline W12 & 3.52 & 38 & 38 & 41 & 23 & 11 \\
\hline K4 & 3.72 & 44 & 43 & 36 & 5 & 18 \\
\hline K2 & 3.92 & 38 & 48 & 36 & 34 & 37 \\
\hline K5 & 3.92 & 48 & 48 & NC & 25 & 5 \\
\hline K9 & 4.10 & 50 & 53 & 55 & 14 & 31 \\
\hline K9a & 4.20 & 50 & 56 & 46 & 17 & 56 \\
\hline $\mathrm{K} 2 \mathrm{a}$ & 4.27 & 44 & 58 & 39 & 7 & 21 \\
\hline $\mathrm{nb}=25$ & & & & & $15 \pm 9$ & $18 \pm 12$ \\
\hline
\end{tabular}

Asterisks $\left(^{*}\right)$ identify cells that were antidromically activated from the predorsal bundle. Calculation of cell location is given in Materials and Methods. Note that fixation cells are placed at $0 \mathrm{~mm}$, but in our analysis of the spatial distribution of activity (Fig. 11), we have randomly assigned these cells to a zone $0-0.5 \mathrm{~mm}$ long. GPE $\mathrm{Estim}, 0$ ptimal amplitude of the cell $=$ amplitude of the stimulation-evoked horizontal gaze saccade. $\mathrm{GPE}_{0 \mathrm{msgs}}$ Optimal amplitude of the cell based on a calculation of the GPE at which peak firing frequency occurs during multiple-step gaze shifts

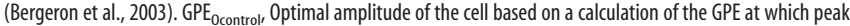
firing frequency occurs during single-step gaze shifts. Gl, Gaze shift interrupted by stimulation; NC, value not calculated because of insufficient data at different GPEs; nb, number.

was isolated from ground (S88, PSIU 6; Grass, Quincy, MA). The antidromic nature of the evoked spike was determined according to the criteria described by Lipski (1981). Cells identified as tectoreticular neurons are indicated in Table 1.

As described in our previous studies (Munoz et al., 1991b; Bergeron and Guitton, 2000, 2002; Bergeron et al., 2003), but contrary to Peck (1987), we did not encounter the typical burst cells frequently observed in the monkey's SC, which have closed movement fields and a strong burst with little preburst, or prelude, activity (Munoz and Wurtz, 1995a) (for review, see Moschovakis et al., 1996; Scudder et al., 2002).

Recall that, in this study of the gaze motor map of the SC, we restricted our recordings to the zone encoding horizontal gaze shifts. This was determined by selecting those sites where electrical stimulation produced nearly horizontal saccadic gaze shifts. For example, stimulating the site where cell $\mathrm{K} 2$ was recorded produced the gaze shifts shown in Figure $1 \mathrm{~A}$. Figure $1 B$ shows the vectors evoked at the sites where we recorded neurons that form the database for this paper. Cells K2, K2A, and K10, used as major examples in this paper, are specifically identified.

We also recorded the activity of collicular fixation neurons (SCFNs) in the fixation zone of the rostral SC. We described the characteristics of cat SCFNs previously (Munoz and Guitton, 1991; Bergeron and Guitton, 2000, 2002), which provided the following criteria, used here, to identify these cells: (1) SCFN tonic activity increases when the cat actively fixates a visible target; (2) this activity persists when the target momentarily disappears but the animal maintains fixation in the dark; (3) the tonic activity pauses for the duration of primarily contralateral single-step gaze saccades; (4) at the end of primarily contraversive gaze saccades to a target, the tonic activity of most cells exceeds that at the start, even if the target has disappeared and the saccade is made in the dark; and (5) some cat SCFNs are tectoreticular neurons.

Data analysis. During the experiments, action potentials were con-
A

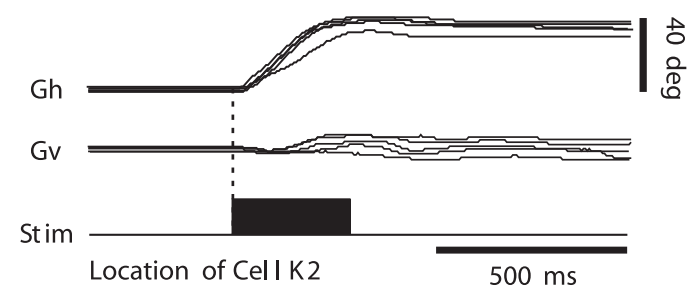

B

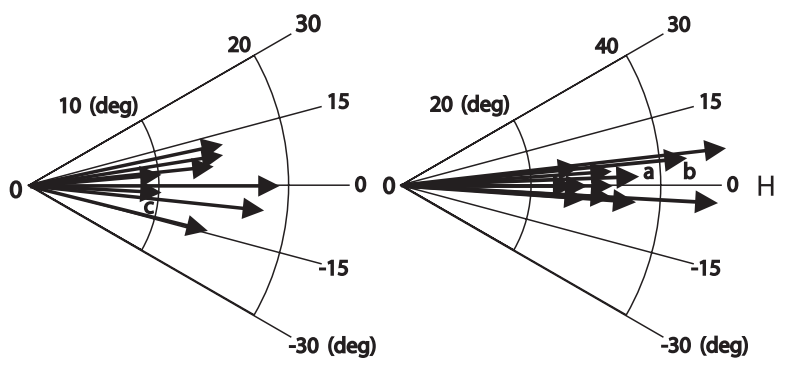

a Cell K2

b Cell K2a

c Cell K10

Figure 1. Gaze saccades evoked by stimulating the $S C . A, A$ train of rectangular cathodal pulses ( $300 \mathrm{~Hz} ; 300$ msec duration) was delivered to the left SC at the position of cell K2. Stimulation evoked $38^{\circ}$ right horizontal gaze movements. $B$, Representative, nearly horizontal, optimal vectors evoked at the sites where we recorded neurons in this study. Vectors $a, b$, and $c$ in polar coordinates result from stimulation at the location of cells K2, K2a, and K10, discussed extensively in the text. Gh, Gaze horizontal; Gv, gaze vertical; Stim, stimulus; H, horizontal.

verted to logic pulses via a time-amplitude window discriminator (BAK Electronics, Mount Airy, MD). The target, eye and gaze positions, cell activity given by the logic pulses, light offset, and duration of dark period were stored on DAT tape (RD-200T; Teac, Montebello, CA) for off-line analysis. The gaze and head coils gave signals proportional to eye and head positions in space, respectively. [Horizontal gaze $(\mathrm{G})=$ horizontal eye position in space $=$ horizontal eye in head $(E)+$ horizontal head in space $(\mathrm{H})$.] Information about calibration procedures for our search coil in magnetic-field technique (Robinson, 1963) have been reported previously (Guitton et al., 1984). In off-line analysis, using the data stored on DAT tape, we calculated $\mathrm{E}$ from $\mathrm{G}$ and $\mathrm{H}$. Movement traces were filtered at $1 \mathrm{kHz}$ and digitized at $2 \mathrm{kHz}$ with data acquisition software and subsequently analyzed with MatLab (MathWorks, Natick, MA). The start and end of saccadic eye and gaze movements were determined by a velocity threshold corresponding to velocity of $25^{\circ} / \mathrm{sec}$. For head movements, the criterion was that velocity $=15 \% \mathrm{sec}$. In brake trials, we measured gaze plateau duration as follows. The beginning of the plateau corresponded to when the velocity of the first gaze saccade dropped to $25 \% \mathrm{sec}$. The end of the plateau was when the velocity of the corrective saccade reached $25 \%$ sec.

During an experiment, contact with a cell was frequently lost, particularly during brake trials, and this imposed serious limitations on the number of cells per cat available for analysis. For a cell to be retained for a comparative analysis of brake and control trials (Table 1), we required that there be at least four large $\left(\approx 50^{\circ}\right)$ control single-step gaze shifts and at least four successful trials of the same intended amplitude in the brake condition. This was in addition to the data on the discharge characteristics of the cell during multiple-step gaze shifts that we needed to accurately localize the cell on the SC map (see Calculation of the spatial distribution of activity on the SC map). We obtained an acceptable set of data on control and brake trials for 20 cells on the motor map in three cats (Table 1): 13 cells in cat K, 1 cell in cat S, and 6 cells in cat W. An acceptable number of trials was obtained for five SCFNs in the rostral SC 
of two cats, three cells in cat $\mathrm{N}$, and two cells in cat $\mathrm{W}$. We described previously (Bergeron and Guitton, 2000, 2002; Bergeron et al., 2003), in relation to variations in GPE during multiple-step gaze shifts, the discharge characteristics of 3 of 5 of our SCFNs and 15 of 20 of our cells on the motor map. The new cells in this report are marked NC in the $\mathrm{GPE}_{\text {Omsgs }}$ column in Table 1 .

In cat $\mathrm{W}$, one SCFN and three of the six cells that we studied in detail on the motor map were tectoreticular neurons identified antidromically as projecting to the reticular formation (Table 1). Note that there have been very few studies of the discharge properties of identified collicular output neurons in the alert animal. Our identification of these neurons provides important insight into the type of signals sent by the SC to downstream pontine circuits. The exact proportion of cells that were collicular output neurons is difficult to state because of the inherent experimental difficulties in identifying tectoreticular neurons in the head-free cat, namely, variability in the long-term reliability of the stimulating electrode as well as the possibility that a cell in the SC sends its axon through a part of the predorsal bundle far from the tip of the stimulating electrode (Munoz et al., 1991b; Bergeron and Guitton, 2000, 2002; Bergeron et al., 2003). We believe that we underestimated the percentage of tectoreticular neurons.

Mean firing frequency during a gaze plateau was obtained by counting the number of spikes (logic pulses) in the gaze plateau and dividing by the duration of the plateau. The number of spikes during a gaze shift was obtained in the period $10 \mathrm{msec}$ before gaze shift onset to $10 \mathrm{msec}$ before end. Other specific measures are given in Results. Spike density histograms were generated in most cases by substituting for each spike a Gaussian function with a width of $10 \mathrm{msec}$ (MacPherson and Aldridge, 1979; Richmond et al., 1987) and then summing all of the Gaussians together to generate a continuous function in time (spike density histogram). However, in those analyses in which we needed to calculate short latencies, we reduced the Gaussian width to $4 \mathrm{msec}$ (see Fig. $3 B, C$ ) to increase the low-pass filtering cutoff frequency.

We showed in previous publications that, in cat SC, tectoreticular neurons encode GPE (Munoz et al., 1991a,b; Bergeron and Guitton, 2000, 2002; Bergeron et al., 2003). To examine this relationship in the present situation, we aligned, for any one cell, the gaze shifts in control trials and corresponding spike density histograms, on different values (in $1^{\circ}$ steps) of GPE in the range from the largest gaze shift amplitude available $\left(\sim 50^{\circ}\right)$, in at least four trials, to $0^{\circ}$. We then calculated the mean firing frequency in each $\Delta \mathrm{GPE}=1^{\circ} \mathrm{bin}$, which permitted us to generate a phase plane plot of firing frequency versus GPE. We also calculated phase plane plots for brake trials by cutting out the plateau (constant GPE) portion of the perturbed gaze trajectory and the corresponding segment of the firing-frequency profile. We analyzed separately the plateau discharges. To compare phase plane plots in the control and brake trials, we used the two-sample Kolmogorov-Smirnov goodness-of-fit hypothesis test ( $\mathrm{K}-\mathrm{S}$ test).

Calculation of the spatial distribution of activity on the SC map. To examine how the spatial distribution of gaze saccade-related activity varied with GPE across our population of cells on the SC map (see Fig. 11), we used the following approach. First, we assumed an example gaze shift of $40^{\circ}$. Second, for each cell, we obtained from the mean phase plane plot of firing frequency versus GPE for single-step gaze shifts, the firing frequency at its peak $\left(\mathrm{GPE}_{\text {Ocontrol }}\right)$ and at GPE of 40,10 , and $0^{\circ}$. We chose $10^{\circ}$, because cells at this location are physically about midway between the fixation zone and the site encoding $40^{\circ}$ gaze shifts. We normalized the frequency at each GPE by dividing by the peak response of the cell, at its optimal GPE. Third, for each value of GPE, we plotted the normalized firing frequency of each cell versus its position on the map (see next paragraph). Fourth, we fitted a Gaussian function to all of the data points (Bergeron and Guitton, 2002).

In this paper, we calculated the position of a cell, $P$ (in millimeters), on the motor map, using the following equation: $P=0.66 \times$ (preferred GPE of the cell) ${ }^{0.46}$ Munoz et al., 1991b), where the preferred or optimal GPE could be either of the following two values: (1) $\mathrm{GPE}_{\mathrm{Ostim}}$, the mean gaze amplitude obtained by stimulating the recording site, or (2) $\mathrm{GPE}_{\mathrm{Omsgs}}$, for multiple-step gaze shifts, the GPE at which peak firing frequency occurs in the phase plane plot of firing frequency during an intersaccade gaze plateau versus the GPE of the plateau (Bergeron and Guitton, 2000, 2002; Bergeron et al., 2003).

We showed previously (Paré et al., 1994; Bergeron et al., 2003) that a gaze saccade evoked by stimulating a given site has, on average, a vector similar to that deduced from the discharge properties, of a cell recorded at that site. Indeed, for our cells on the motor map, the relationship between $\mathrm{GPE}_{\mathrm{Ostim}}$ and $\mathrm{GPE}_{\text {Omsgs }}$ (Table 1) was excellent: $\mathrm{GPE}_{\mathrm{Ostim}}=$ $\left(0.99 \times \mathrm{GPE}_{\text {Omsgs }}\right)-4.62 ; r=0.96$. In Bergeron et al. (2003), we also verified that $\mathrm{GPE}_{\text {Omsgs }}$ was similar to that obtained by measuring the retinotopic location of the optimal visual response of a cell. Note that another estimate of the position of a cell could be obtained in the present experiments from the GPE at which peak firing frequency occurs in the phase plane plot (see Fig. 10) of firing frequency versus GPE for singlestep control gaze shifts (Table $1, \mathrm{GPE}_{\text {Ocontrol }}$ ). For our cells, the equation was as follows: $\mathrm{GPE}_{\text {Ocontrol }}=\left(0.78 \times \mathrm{GPE}_{\text {Omsgs }}\right)+0.22 ; r=0.93$. Thus, the optimal GPE of a cell, obtained at peak discharge during control gaze shifts, was linearly related to, but $\sim 20 \%$ less than, the optimal GPE calculated from multiple-step gaze shifts. We cannot explain this difference. In multiple-step gaze shifts, the estimate of the GPE of a cell at peak firing frequency may be more accurate than for single-step control gaze shifts, because the calculation is based on static quantities, and there is no need to be concerned with time delays between cell firing and GPE. Indeed, the GPE of a given gaze plateau is constant, and the plateau lasts generally $\sim 125$ msec; mean firing frequency in the plateau can be estimated reliably by counting spikes.

We had data from 24 of 25 cells in Table 1 from which to estimate the pattern of discharge on the SC map during large single-step control and braked gaze shifts. (We had no control gaze shifts of the appropriate amplitude for cell W113C.) We could not calculate $\mathrm{GPE}_{\mathrm{Omsgs}}$ for five cells on the motor map. We estimated their position in Figure $11 G-I$ using GPE $\mathrm{Ostim}_{\text {. }}$.

An accurate estimate of the spatial pattern of discharge on the collicular map benefits from data from as many cells as possible. Therefore, to improve our estimate in the control condition, we used a group of 10 other cells, which we had studied during multiple-step gaze shifts, and for which we had an acceptable number of single-step control gaze shifts. We had no brake data for these 10 extra cells. Seven of them were studied in cats $\mathrm{M}$ and $\mathrm{P}$, not used in the brake studies. Seven (not the same as the seven in the preceding sentence) of the 10 extra cells were SCFNs (cells M54, M54a, M56, N26, N27a, P47a, and P48b) for which we had a mean of $11 \pm 5$ trials (minimum, 4 ; maximum, 19). Three of the 10 extra cells were on the motor map (cells K7a, M57a, and M58a) for which we had a mean of $44 \pm 26$ trials (minimum, 19; maximum, 64). The 10 extra cells could be localized on the map using either $\mathrm{GPE}_{\mathrm{Ostim}}$ or $\mathrm{GPE}_{\mathrm{Omsgs}}$. Thus, to estimate the position of a cell on the collicular map, we used both $\mathrm{GPE}_{\text {Omsgs }}$ (see Fig. $11 A-C$ ) and $\mathrm{GPE}_{\text {Ostim }}$ (see Fig. $11 D, E$ ).

\section{Results}

Our population of cells in two cats sampled well that part of the collicular map that included the rostral fixation zone and a band along the horizontal meridian lying $1.9-4.3 \mathrm{~mm}$ caudal to it (Table 1). The precise extent of the fixation zone is unknown-it is thought that cells with SCFN-like properties can be recorded in a region of the rostral $\mathrm{SC} \sim 0.5-0.7 \mathrm{~mm}$ long in the rostrocaudal direction (Munoz and Wurtz, 1995b; Anderson et al., 1998). We could not objectively assign SCFNs to specific positions within that region. As we will see in the ensuing sections, there was an excellent link between the discharge properties of a cell and its location on the motor map.

\section{Compensation for interrupted gaze shifts}

Before considering the responses of SC neurons to gaze shift perturbations and the implications of these results for the feedback control of gaze saccades, it is important to show that our cats actually compensated for the brake-induced gaze perturbation. Figure $2 A-D$ shows typical examples of control and perturbed gaze shifts, respectively, both made in the dark by cat $\mathrm{K}$ to the 
A
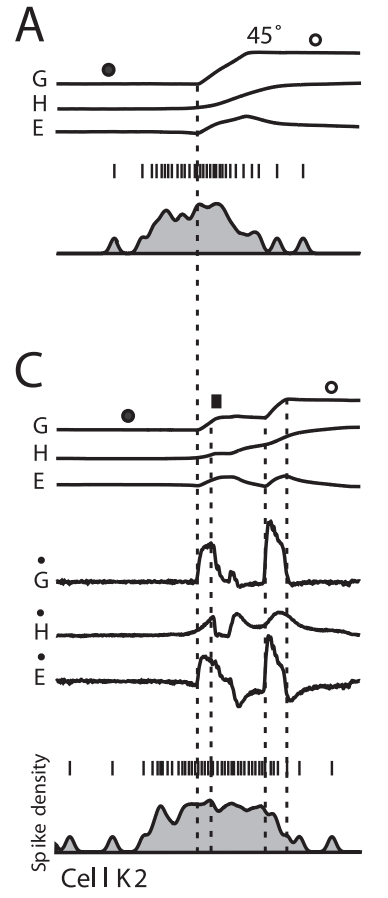

E

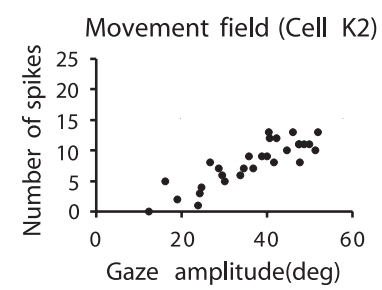

B

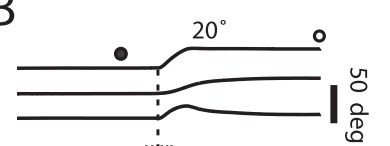

II,III
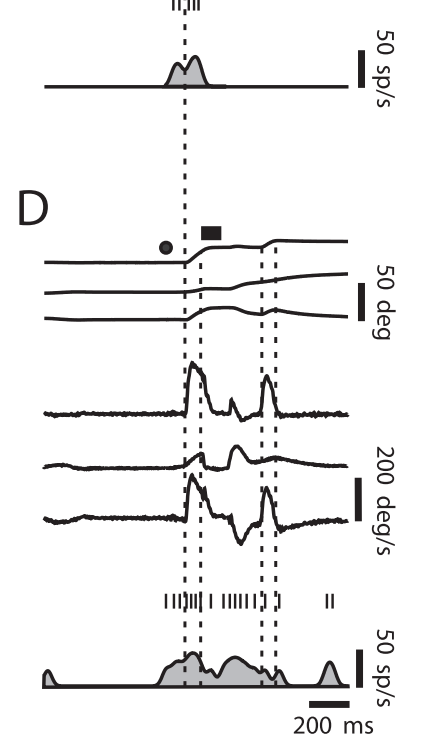

F

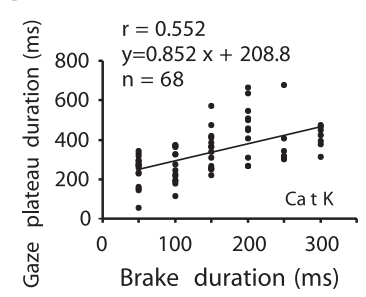

Figure 2. Coordinated eye-head movements in cat Kand associated discharge of typical cell $\mathrm{K} 2$ in caudal left $\mathrm{SC}$ at the $48^{\circ}$ location. $A$, Horizontal gaze $(\mathrm{G})$, eye $(\mathrm{E})$, and head $(\mathrm{H})$ positions during a large control gaze shift of amplitude $45^{\circ}$. Upward deflections indicate rightward movements. The bottom trace shows neuron firing frequency given as a spike density function. Above are vertical tic marks representing action potentials. Closed and open circles above $G$ trace indicate offset and onset of ambient lighting. This and all of the other illustrated gaze shifts were made in complete darkness. $B$, Discharge of cell during a control gaze shift of small amplitude $\left(20^{\circ}\right)$. Note lower firing frequency compared with large movement. $C$, Discharge of cell during a perturbed gaze shift of same intended amplitude $\left(45^{\circ}\right)$ as control. Brake duration, 50 msec, indicated by thick bar over $\mathrm{G}$ trace. Vertical dotted lines indicate, respectively from left to right, gaze onset, brake onset, end of the gaze plateau, and end of the corrective gaze saccade. $\dot{\mathrm{G}}, \dot{\mathrm{E}}$, and $\dot{\mathrm{H}}, \mathrm{Gaze}$, head, and eye velocity. Note that cell continued discharging at same frequency during gaze plateau. D, Discharge of cell during a perturbed gaze shift of small amplitude $\left(20^{\circ}\right.$ as in $B$ ). Brake duration, $100 \mathrm{msec}$. Activity continued during gaze plateau, but there was a transient suppression just after head perturbation. $E$, Relationship between number of spikes during single-step control gaze shifts and gaze amplitude. The cell had an open-ended movement field. F, Relationship between gaze plateau duration and brake duration. Correlation is not strong, because eye and head moved in opposite directions after brake release and so lengthened gaze plateau relative to brake duration.

remembered target location. The 45 and $20^{\circ}$ control gaze saccades in Figure 2, $A$ and $B$, respectively, are composed of the now well known pattern of coordinated eye and head motion (for review, see Guitton, 1992). When gaze reached the target, the eye, driven by the vestibulo-ocular reflex (VOR), moved in a direction opposite to head motion to stabilize eye position in space. The perturbed gaze shifts in Figure 2, $C$ and $D$, are of the same intended amplitude as those in Figure 2, $A$ and $B$, respectively, but were interrupted by a short $50 \mathrm{msec}$ brake (short heavy line above gaze trace) that immobilized the head shortly after its onset. The eye saccade stopped soon after head velocity declined to zero, and

during the ensuing period of head immobilization, the eye remained immobile in the orbit. For our entire data set on braked movements (Table 1), the mean time between the onset of the brake-induced head deceleration and when eye velocity became zero (i.e., the onset of the gaze plateau) was $44.5 \pm 18.6 \mathrm{msec}$. After brake release, the head resumed its movement, and an ocular counterrotation compensated for initial head motion to stabilize gaze, resulting in a gaze plateau of $\sim 250 \mathrm{msec}$ duration, $\sim 200$ msec longer than brake duration.

The considerable variability in plateau duration for a given brake duration is illustrated for cat $\mathrm{K}$ in Figure $2 \mathrm{~F}$ by the linear regression line: plateau duration $=[0.85 \times($ brake duration $)]+$ 209; $r=0.55$. For cat $\mathrm{W}$, this relation was as follows: plateau duration $=[0.82 \times($ brake duration $)]+201 ; r=0.61$. The fact that gaze plateaus were longer than brake duration can be explained as follows: (1) the rapid head deceleration truncated the saccade by suppressing the discharge in the burst generator (Cullen and Guitton, 1996); (2) this placed the system in the slow-phase mode of the VOR, producing an eye rotation that compensated for initial head motion; and (3) as the head rotation continued, the eye was driven in the contralateral direction, thereby prolonging gaze plateau duration. Eventually, with the cat still in the dark, a corrective eye saccade was triggered, which, together with head motion, brought gaze onto the target zone. No corrective saccades that preceded head release were ever seen.

The accuracy of gaze shifts in the control and perturbed trials was indistinguishable. We define gain as the ratio of overall gaze shift amplitude-measured between gaze shift onset and $10 \mathrm{msec}$ before ambient lighting resumed-in brake trials divided by target offset angle relative to starting gaze position. We found that, in cat $\mathrm{K}$, the mean gains $\pm \mathrm{SD}$ of control and perturbed trials were $0.99 \pm 0.057$ and $0.96 \pm 0.050$, respectively. In cat $\mathrm{W}$, these values were $0.94 \pm 0.089$ and $0.94 \pm 0.086$, respectively. In cat $S$, these values were $0.86 \pm 0.139$ and $0.86 \pm 0.135$, respectively. Clearly, in all of the cats, the corrective saccades compensated in the dark for the perturbations and brought gaze with normal accuracy to the remembered location of the target.

In the following sections, we will first present the typical discharge patterns of collicular cells, and then an overview and comparison of the population responses in the control and brake trials.

\section{Cell discharges on the motor map during large control and perturbed gaze shifts}

Figure 2 shows an example of a typical cell (K2) recorded in cat K in the caudal left SC where large gaze shifts are encoded. Electrical microstimulation delivered to the recording site of this cell induced, on average, a $38^{\circ}$ nearly horizontal rightward gaze shift. Analysis of its discharge during multiple-step gaze shifts placed cell $\mathrm{K} 2$ at the $48^{\circ}$ location on the horizontal meridian of the SC map (Table 1) (Bergeron et al., 2003). In the present report, we will assume that the cell is at $48^{\circ}$. Note that small uncertainties in its location are not critical in terms of distance on the SC map, because the isoamplitude lines in the caudal SC are close together.

The cell had long-lead activity that preceded the rightward $45^{\circ}$ gaze shift (Fig. $2 \mathrm{~A}$ ) by $>200 \mathrm{msec}$. The activity gradually increased until gaze saccade onset, and there was no distinct burst preceding the gaze shift, although it was about the optimal amplitude for neurons at this recording site. The lack of a clear burst is frequently observed in cat tectoreticular neurons during gaze shifts in the dark (Munoz et al., 1991b). For a smaller $20^{\circ}$ gaze shift, the cell had a shorter, lower-frequency discharge (Fig. $2 B$ ). Plotting the total number of spikes in a discharge (see Materials 
A

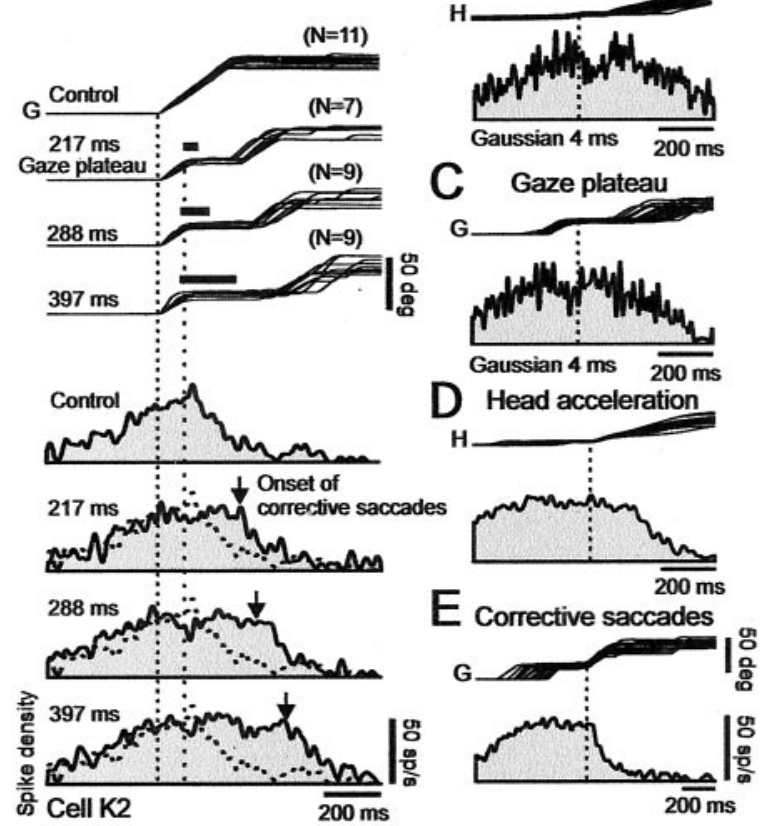

Figure 3. Effect of perturbing head (and therefore gaze) trajectories on discharges of cell $\mathrm{K} 2$ in caudal $\mathrm{SC}$ at $48^{\circ}$ location. $A$, Gaze (G) traces show, from top to bottom, control and three selected brake conditions with mean gaze plateau durations of 217, 288, and 397 msec, respectively. Trials were also selected such that plateaus are about at a fixed position relative to the target, at $\mathrm{GPE}=25 \pm 5^{\circ}$. Number of superimposed trials in each condition is indicated at top right of each group of traces. The spike density histograms that correspond to each condition are shown sequentially below the gaze traces. All of the traces aligned on gaze onset (left vertical dotted line). Right vertical dotted line indicates start of brake-induced plateaus. Thick bars over plateaus indicate average duration of brake. Vertical down arrow in each spike density histogram indicates average time of onset of corrective gaze saccades. Note that firing frequency remained about constant during gaze plateau, and discharge duration increased with plateau duration. $B$, Effect of head brake on activity. Top trace shows the superimposed head $(H)$ trajectories for all 35 brake trials in $A_{t}$ aligned on onset of head deceleration. Bottom trace shows associated weak transient depression in the average spike density histogram. C, Effect of head brake on activity during gaze plateau. Transient depression was over at start of plateau. D, Effect of head acceleration. Same as in $B$ but with head traces now aligned on onset of head acceleration after brake release. Activity was not directly affected by head acceleration. E, Activity linked to corrective gaze saccades. Traces aligned on onset of corrective gaze saccades. Note the absence of saccade-related bursts, and that, during corrective saccades, activity decreased monotonically to near zero at gaze end. Spike density histograms were obtained using $10 \mathrm{msec}(A, D, E)$ and $4 \mathrm{msec}(B, C)$ Gaussian filters. position at $\sim 50^{\circ}$ on the motor map, the firing frequency of cell $\mathrm{K} 2$ reached a maximum at about the onset of the $50^{\circ}$ control gaze shifts. The firing rate held about steady during the early portion of this gaze shift and then, when GPE reached $\sim 25^{\circ}$, began declining rapidly. Regarding the trials in which head brakes were applied, note that, as expected, the firing-frequency profiles in the control and perturbed trials were the same until the beginning of the brake-induced plateaus. The important observation for all of the brake trials is that the cell continued to discharge throughout all of the brake-induced plateaus, even if a plateau lasted $400 \mathrm{msec}$. Furthermore, the discharge declined to zero at the end of both the control and perturbed gaze shifts. These observations imply that the SC was receiving feedback signals, indicating both that the gaze shift had been interrupted and when it was completed.

In some, not all, head brake trials, a slight transient suppression of the discharge of $\mathrm{K} 2$ occurred, corresponding to the onset of head deceleration (Fig. 2D shows one example). As a result, for this cell, there was a shallow and brief notch in the spike density histogram when all 35 brake trials in Figure $3 A$ were aligned on the onset of head deceleration (Fig. 3B). Activity after the notch returned to the prebrake value before the onset of the gaze plateau (Fig. 3C). There was no increase in activity either when the head reaccelerated after brake release or before the corrective saccade (Fig. $3 D, E$, respectively). An overview of the discharges of all of the cells in the caudal SC will be presented later.

Figure 4 shows the discharge characterand Methods) for all of the amplitudes shows that the cell had an open-ended movement field (Fig. 2E). This cell is typical of those described by Munoz et al. (1991b).

Interestingly, the cell activity continued during the brakeinduced gaze plateaus. In the perturbed $45^{\circ}$ gaze shift (Fig. 2C), the firing frequency during the plateau was almost the same as that just preceding the plateau. For the smaller movement (Fig. 2D) (about one-half of the amplitude of the optimum for that recording site), there was a transient suppression of activity after the brake, and activity resumed again during the plateau. In Figure 2, both $C$ and $D$, firing frequency gradually decreased to near zero during the corrective gaze saccade, similarly to that occurring toward the end of the control movements. Note that the constant tonic firing frequency during the gaze plateau in Figure $2 C$ was modulated neither by the head motion that resumed after the brake nor by the ocular counterrotation (VOR) that accompanied it.

Figure $3 \mathrm{~A}$ generalizes these observations for cell $\mathrm{K} 2$ by showing a series of control and perturbed large $50^{\circ}$ gaze shifts, the latter selected and classified according to different plateau durations. All of the trials were selected from the database such that plateaus occurred at an approximately fixed gaze position relative to the target, specifically at GPE of $25^{\circ} \pm 5$. In agreement with its istics of another cell, $\mathrm{K} 10$ in cat $\mathrm{K}$, located at $\sim 20^{\circ}$ (based on $\mathrm{GPE}_{\text {Omsgs }}$ ) (Table 1) in the middle zone of the right SC. Figure $4 \mathrm{~A}$ shows that the discharge preceded small $10^{\circ}$ gaze shifts, as expected from the position of the cell on the map. We had no control $20^{\circ}$ gaze shifts for this cell. The discharge pattern of cell K10 during large gaze shifts was strikingly different from that of cell $\mathrm{K} 2$ in both control and brake trials. For example, in the former, the cell had little activity preceding $50^{\circ}$ leftward control gaze shifts (Fig. 4C). Rather, for these large gaze shifts, the peak firing frequency was delayed relative to gaze shift onset, a phenomenon typical of cells in the middle and rostral cat SC (Munoz et al., 1991a,b). Plotting the total number of spikes in a burst for all of the amplitudes shows that the cell had an open-ended movement field (Fig. 4B). (Although we did not obtain control single-step gaze shifts of the optimal amplitude for this cell, we could calculate the optimal GPE using both stimulation and multiple-step gaze shifts.)

Figure $4 C$ compares the discharge of the cell during control and perturbed large gaze shifts, the latter selected and classified according to different plateau durations. We show trials with plateaus at GPE of $\sim 20^{\circ}$, close to the preferred GPE of the cell. Note that, in all of the brake trials, the activity profile of the cell before the brake-induced plateaus was the same as in the control 


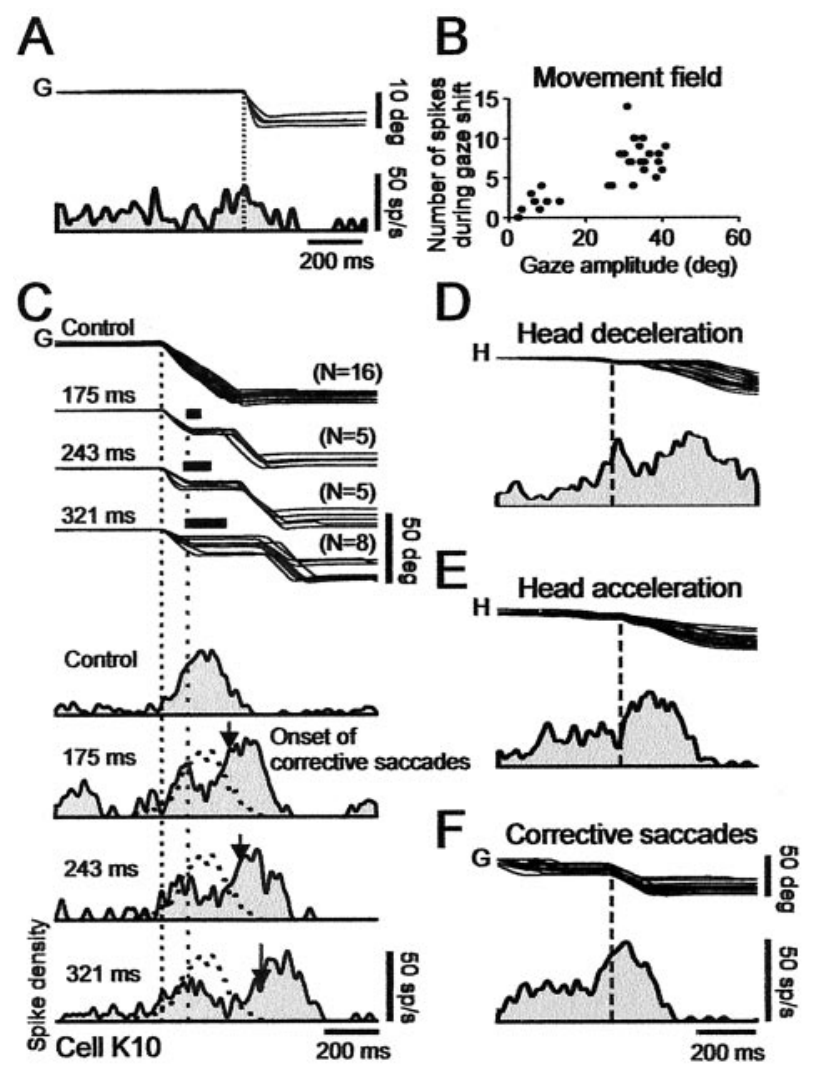

Figure 4. Discharge of cell $\mathrm{K} 10$, in middle right $\mathrm{SC}$ at $19^{\circ}$ location, during control and perturbed gaze shifts. $A$, Ten degree gaze (G) shifts and associated cell discharge. $B$, Number of spikes in a discharge versus gaze saccade amplitude. Cell discharges for all of the gaze shifts larger than its $19^{\circ}$ optimum (i.e., it has an open-ended movement field). C, Discharge during large gaze shifts. Top traces show superimposed gaze trajectories in control trials (top gaze traces) and different brake trials selected to have plateaus at about a fixed GPE of $\sim 20^{\circ}$. The illustrated gaze traces have gaze plateau durations with means of 175, 243, and $321 \mathrm{msec}$. Traces below gaze trajectories show the spike density waveforms associated with the different plateau durations. All of the traces aligned on gaze onset (left vertical dotted line). Onset of plateaus indicated by right vertical dotted line. Thick bars over plateaus indicate average duration of brake. In control trials, peak discharge lagged onset of large gaze saccades. For perturbed trials, arrows on spike density histograms indicate average time of onset of corrective gaze saccades. After brake onset, firing frequency first declined and then increased before corrective gaze saccade. Overall discharge duration increased with plateau duration. $D$, Effect of brake. Superposition of all of the brake trials in C, aligned on head deceleration (vertical dotted line). The discharge was suppressed shortly after head $(H)$ decelerates. E, Effect of brake release. Same traces as in $D$, now aligned on onset of the head acceleration (vertical dotted line) that followed brake release. Activity increased after the head was released. F, Burst-like activity before corrective gaze saccades. Same traces as in $D$ aligned on onset of $\sim 30^{\circ}$ corrective gaze saccades (vertical dotted line). Note that peak discharge lagged saccade onset, compatible with movinghill hypothesis. All of the spike density profiles were obtained using $10 \mathrm{msec}$ Gaussians filter.

movements. In all of the brake trials, there was a sharp decrease, relative to control, in firing frequency that began just after the brake-induced head deceleration (Fig. 4D) and gradually dropped to zero during the longest plateaus ( $C$, bottom trace). Accordingly, the time at which activity reached a minimum increased with plateau duration. Activity increased after the minimum, in relation to the time of occurrence of head acceleration (Fig. $4 E$ ). Firing frequency reached its maximum at about the middle of the $30^{\circ}$ corrective saccades (Fig. $4 F$ ). (The location of peak discharge will be considered in more detail subsequently in relation to Fig. 11.)

The firing-frequency profile of cell K10 contrasts with that of cell $\mathrm{K} 2$ recorded more caudally in that the latter maintained a
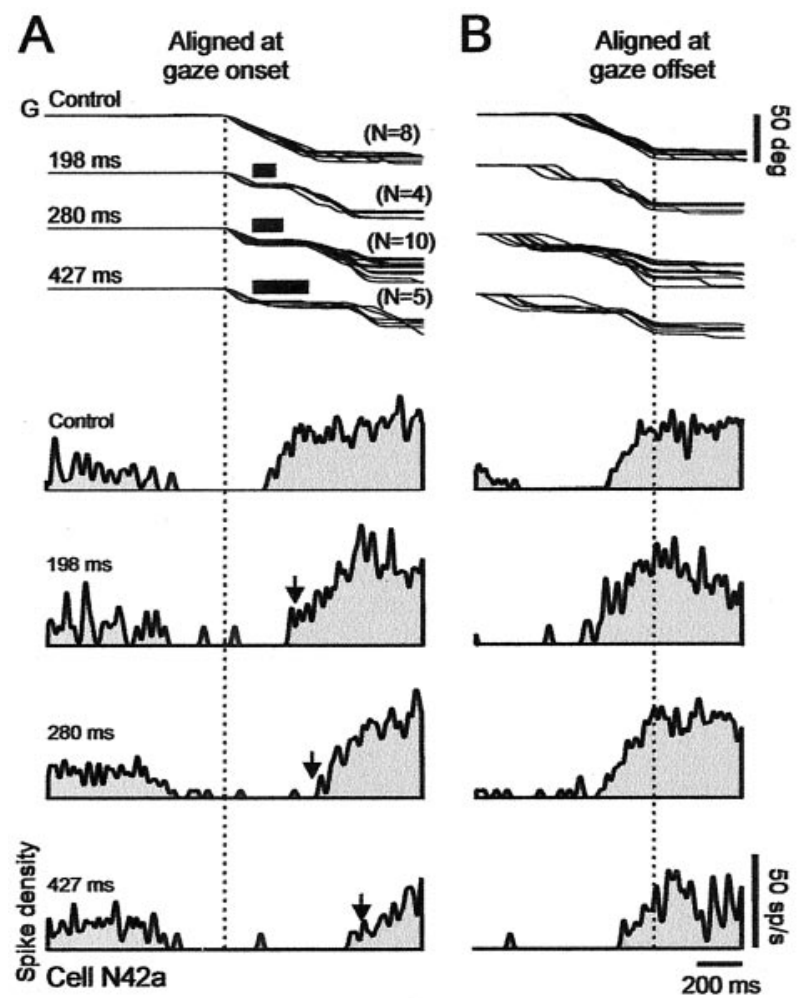

Figure 5. Discharge of collicular fixation cell N42a during control and perturbed trials. Prolongation of pause in activity during perturbed gaze $(\mathrm{G})$ shifts. $A$, Traces aligned on onset of gaze shifts. Superimposed gaze traces at top of panel show control trials. The three below show brake trials. Traces of brake trials show, from top to bottom, mean gaze plateau durations of 198, 280, and $427 \mathrm{msec}$. Thick bars over plateaus indicate average duration of brake. Bottom four traces in panel show sequentially the spike density histograms associated with the control and different plateau durations. Note that pause in activity increased with plateau duration. Arrows indicate end of plateaus. $B$, Traces aligned on gaze shift end. Note that activity peaks at end in both control and brake trials. Spike density histograms use $10 \mathrm{msec}$ Gaussian filter.

nearly steady tonic discharge during plateaus. Nevertheless, the overall discharge duration of cell K10, measured from gaze shift onset to the end of the second burst, lasted far longer than the duration of the control gaze shift, similar to what we saw for cell K2.

Discharges of fixation neurons during large control and perturbed gaze shifts

Figure 5 shows the activity of a collicular fixation neuron (SCFN N42a) during a large gaze shift made by cat $\mathrm{N}$ in a direction contralateral to the recording site. This cell was located in the rostral pole of the right SC. The cell had a low discharge at the start of the trial (Fig. 5A) when the cat faced the blank opaque barrier. The firing of the cell paused completely, shortly after target presentation, and resumed during the gaze shift.

The pause clearly was prolonged when the gaze trajectory was perturbed in midflight, at the time at which the cell was already silent (at GPE of $30^{\circ}$ in this example). In previous studies of natural multiple-step gaze shifts, we found that the first spike after the pause is determined by when gaze arrives at a particular distance from the target, on average when GPE $=13^{\circ}$ (Bergeron and Guitton, 2000, 2002). In the present example, cell N42a began to fire, after the pause, on average at GPE of $\sim 20^{\circ}$ in both control and brake trials. After the pause, the firing frequency gradually increased as GPE decreased, and aligning the gaze shifts on their end (Fig. 5B) showed that firing frequency peaked very close to GPE of 0 in both control and brake trials. Here, we could 
not test whether firing frequency during brake-induced plateaus depended on GPE, as in Bergeron and Guitton $(2000,2002)$, because most brake trials occurred during the pause in activity, and, by extension, we obtained too few brake trials in which gaze was immobilized after the pause in activity had ended.

\section{Overview of population discharges}

In this section, we expand on the examples shown in Figures 3-5 for the purpose of introducing specific properties of the spatiotemporal discharge pattern on the SC map that will be addressed more quantitatively in subsequent sections. Figure 6 compares the discharge patterns during control and brake trials of nine cells (five in cat $\mathrm{K}$ and four in cat $\mathrm{W}$ ) whose positions on the map spanned the distance between the rostral and caudal ends of the SC. This figure and the preceding Figures $2-5$, showing cells K2, K10, and N42a, together illustrate the discharges of 12 of our 25 cells. As in Figures 3-5, we show the firing-frequency patterns during large $\left(\approx 50^{\circ}\right)$ gaze shifts.

Cells in each row of Figure 6 were recorded at about similar locations on the motor map in each cat. We had neither fixation neurons in cat $\mathrm{K}$, nor neurons in the very caudal SC in cat W; all were lost in brake trials. Plateau duration varied from cell to cell, because we retained brake trials only when at least four plateaus of about the same duration and GPE were available for constructing a firing-frequency histogram, and sometimes this could be obtained only for a limited range of plateau durations. An impression of the differences in the activity patterns in the rostral and caudal SC, for both the control and brake trials, can be had by scanning each column in Figure 6 from top to bottom.

The control discharges had similar temporal characteristics to those we described previously on numerous occasions and are compatible with the moving-hill hypothesis. This can be appreciated by considering the timing of the peak discharge relative to gaze shift end, indicated by the length of the short horizontal line just below the gaze traces in each panel. Thus, for caudal cells (bottom of Fig. 6), the peak of the control discharge (left tic mark on horizontal line) occurred close to the onset of the $50^{\circ}$ gaze shifts (Fig. 6A5). In comparison, for rostral cells (top of Fig. 6), the peak of the control activity lagged gaze shift onset (Fig. $6 A 1,2)$, and occurred progressively closer to gaze shift end as the cell lay nearer to the rostral pole of the SC (to be considered in more detail later in Figs. 10 and 11). This discharge pattern-the delayed burst in the rostral SC during large gaze shifts-is necessary (but not sufficient) in the moving-hill hypothesis.

The fact that control and brake trials had similar initial discharge properties until brake onset time follows from the fact that the animals could not predict in which trials the brake would be applied. In general, for large gaze shifts, activity at the start of plateaus, having GPE of $\approx 25^{\circ}$, was as follows: low for cells in the rostral SC at $\sim 0-15^{\circ}$ on the map (e.g., cells K6a and W51), high for cells in the middle SC at $\sim 20^{\circ}$ (e.g., cells K7, W110, and W112d), and low again for very caudal cells at $\sim 60^{\circ}$ (e.g., cell K2a). The latter is particularly striking. The large $\left(\approx 50^{\circ}\right)$ gaze shifts were about optimal for cell K2 located in the very caudal SC, and the firing-frequency profile appropriately rose to a maximum at gaze shift onset and declined as it progressed. Thus, the firing frequency of K2a had declined substantially when the plateau began, and it continued to decline after the brake and for $\sim 50 \mathrm{msec}$ after plateau onset. The discharge held about steady during most of the remaining plateau, as in $\mathrm{K} 2$, and declined to zero at the end of the corrective gaze saccade. The other three cells in the caudal SC (K5, K9, and K9a; not all shown in Fig. 6) had variable rates of decreasing firing frequency during plateaus,
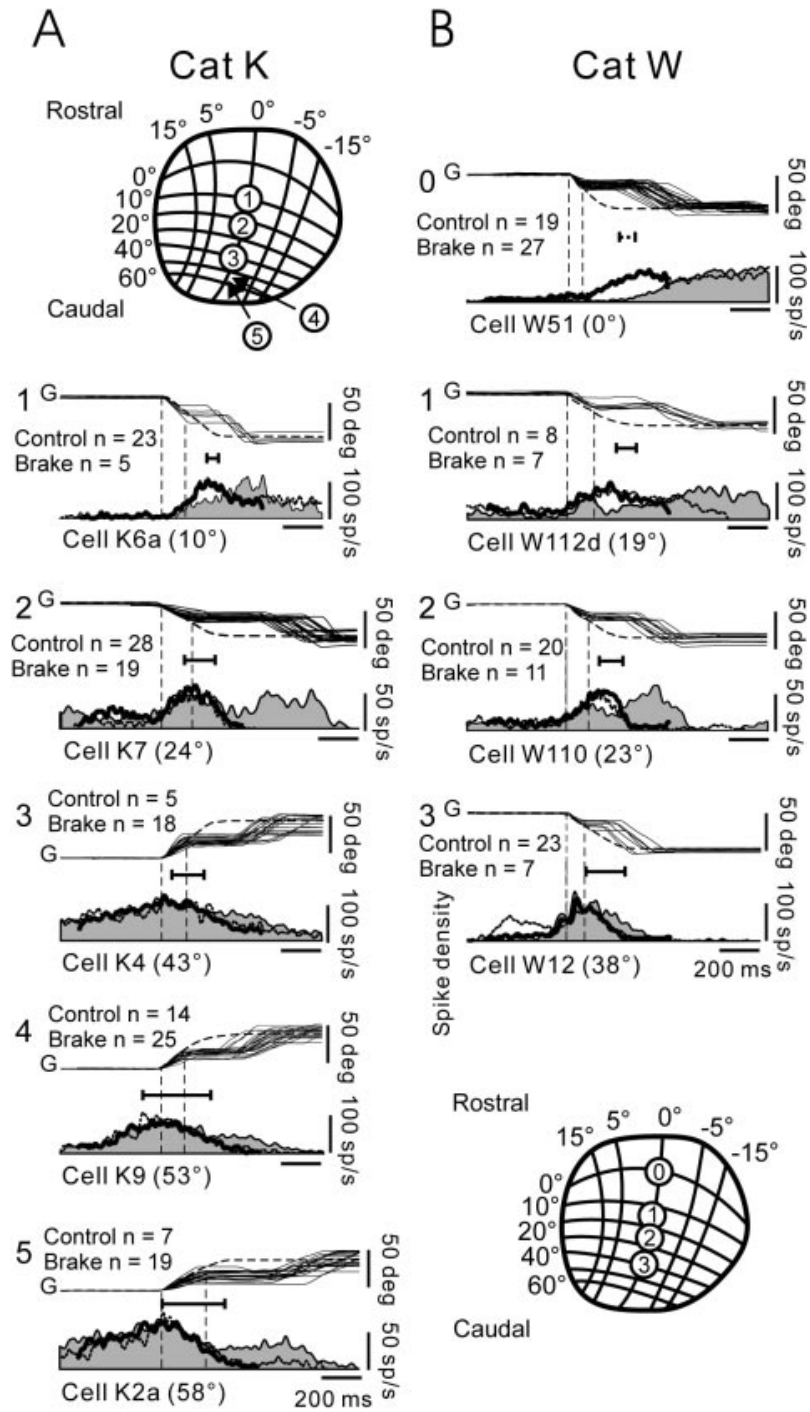

Caudal

Figure 6. Discharge patterns of cells at different rostrocaudal locations on $S C$ map. $A, B$, Data for cats $K$ and W, respectively. $A$, Top; $B$, Bottom, Schema of the gaze motor map in SC (taken from Feldon et al., 1970) with numbers showing the recording sites of cells in corresponding panels of the same column. Cells in each column are arranged from top to bottom according to their rostrocaudal location on the map. Each panel shows the mean control gaze (G) profile (dashed line) and some superimposed brake trials, selected to have about constant plateau duration and GPE, and below, the associated mean firing-frequency profiles. All of the traces are aligned on gaze onset (left vertical dotted line). Onset of plateaus is indicated by right vertical dotted line. The profile for brake trials is filled in, and the control profile is a dotted line. The thick black line in each panel shows the firing-frequency profile that results when plateaus have been cut out and the two ends slid toward each other. For most cells, these are similar to control profiles. Number of control and brake trials in each panel is indicated. Horizontal line below gaze traces in each panel shows time of occurrence of peak discharge relative to the end of the mean control gaze shift; left tic mark on line identifies time of peak discharge; right tic mark is at gaze shift end. See Overview of Population Discharges for additional details.

ranging from no decline in cell $\mathrm{K} 5$ to moderate in $\mathrm{K} 9$ (Fig. 6A4) and $\mathrm{K} 9 \mathrm{a}$.

In comparison, for cells in the middle SC (W112d, W110, K7, and $\mathrm{K} 10$ ), all at about the $20^{\circ}$ location, the firing frequency decreased after the head brake and rose again during the plateau before the onset of the corrective gaze saccade. The rate of decrease in firing frequency varied between cells; compare $\mathrm{K} 7$ and W112d (Fig. 6). As in K10 (Fig. 4), the decrease in firing of most middle-SC cells was interrupted by the increasing discharge, linked to the onset of corrective gaze saccades. 


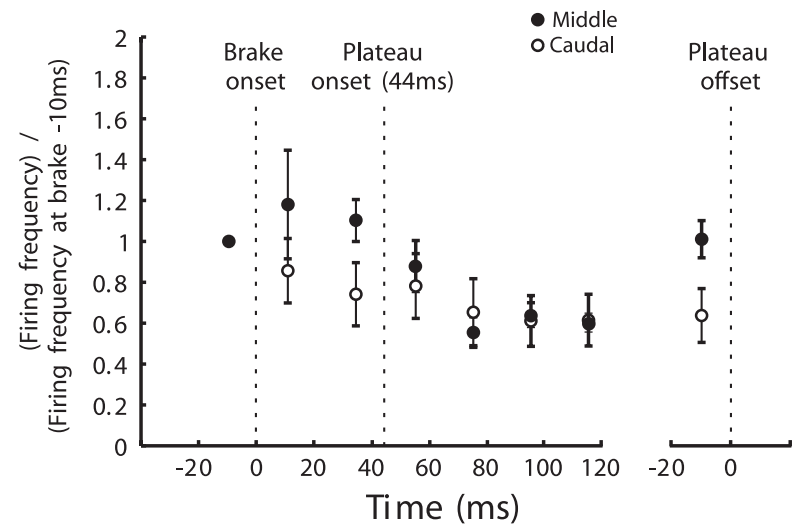

Figure 7. Evolution with time of postbrake mean activity for two populations of cells in the caudal and middle SC, respectively. Caudal cells (open circles), Mean of the mean discharge of each of cells K2, K5, K9, K9a, and K2a. Same for middle cells (dark circles): K7, K10, W112d, and W110. Activity levels for both populations normalized to the mean value in the $20 \mathrm{msec}$ period before brake onset. Mean time between brake and plateau onsets was $44.5 \mathrm{msec}$. The activity levels in the $20 \mathrm{msec}$ period before plateau end are shown to far right. Short vertical lines through each point indicate SE. Note that, for both populations, the activity levels at the start and end of plateaus were equal despite a transient decrease during the plateau. See Activity during Brake-Induced Gaze Plateaus for details.

\section{Activity during brake-induced gaze plateaus}

Despite differences in the postbrake firing-frequency profiles of cells in the middle and caudal SC, the discharge frequency was nearly the same at the start and end of plateaus. To show this, we compare in Figure 7 , for large $\sim 50^{\circ}$ gaze shifts, mean population activity of five cells at about the $50^{\circ}$ location in the caudal SC with that of four other cells at about the $20^{\circ}$ location in the middle SC. Recall that, on average, brake and plateau onsets were separated by $44.5 \pm 18.6 \mathrm{msec}$. We selected brake trials in which plateaus were at least $220 \mathrm{msec}$ long (mean, $255 \pm 62 \mathrm{msec}$ ). We did not analyze activity in the period beyond $120 \mathrm{msec}$ after plateau onset, because, in the middle SC, the rising activity levels that preceded the corrective gaze saccades started $\sim 100$ msec (Fig. $4 F$ ) before plateau end. Figure 7 shows normalized (relative to the mean activity level in the $20 \mathrm{msec}$ period just preceding brake onset) mean population activity levels in the periods: $0-20 \mathrm{msec}$ after brake onset, -20 to 0 msec before plateau onset, and at various time intervals after plateau onset including plateau end.

Activity in middle-SC cells kept increasing after brake onset. As we shall see later in relation to Figure $10, B$ and $C$, this was presumably because of the dominant influence of the continuing eye motion, which, for these large gaze shifts, reduced GPE and increased firing frequency. At about plateau onset, activity in middle-SC cells began decreasing until $\sim 40 \mathrm{msec}$ after plateau onset, followed by a steady discharge (Fig. 7). At plateau end, firing frequency had increased and was equal $(p \geq 0.05)$ to that at plateau onset.

For cells in the caudal SC, activity decreased immediately after brake onset, probably because of the eye motion-related decreasing GPE (see Fig. 10D,E). After plateau onset, activity levels kept decreasing slightly to reach a steady level $\sim 15 \%$ lower at plateau end than at plateau onset (Fig. 7). However, this difference was not significant $(p \geq 0.05$ ). For all of the cells combined across the SC, the mean firing frequency in the $20 \mathrm{msec}$ period at the start of a gaze plateau was $49 \pm 16$ spikes/sec compared with $48 \pm 12$ spikes/sec in the final $20 \mathrm{msec}$ period at the end of a plateau. These means are not significantly different $(p \geq 0.05)$.

On the basis of these results, it follows that, when the plateau portion of every perturbed gaze trajectory was cut out, and the

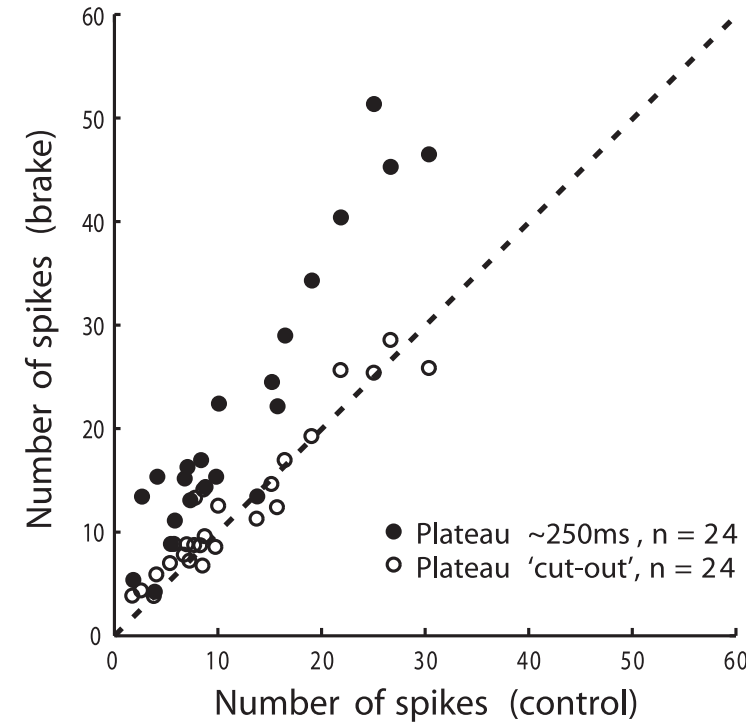

Figure 8. Comparison of number of spikes in control and brake trials (Materials and Methods). Filled circles show data for brake trials selected such that plateau duration was $\sim 250$ msec. Each point represents one cell. Number of cells $n=24$. For all of the cells except two (see Gaze Plateaus Prolong Caudal Cell Tonic Discharges and S( FM Pauses), there were more spikes in brake trials than in control trials. Open circles give number of spikes in the brake trials after the plateaus have been cut out.

two isolated portions were slid toward each other, aligned on gaze onset, and averaged (thick black profiles in each panel of Fig. 6), the reconstituted firing-frequency profiles of 23 of 24 cells (cell $\mathrm{W} 113 \mathrm{c}$ had no control trials) were similar to the associated mean control profiles ( $p \geq 0.05$; K-S test).

\section{Gaze plateaus prolong caudal cell tonic discharges and SCFN pauses}

An assessment of the effect of the brake, applicable to most cells, can be made by counting the number of spikes in the discharge of each cell (Materials and Methods) and comparing the control and brake trials. We chose brake trials in which plateau duration, for each cell, was about as long as control gaze shifts (mean plateau duration, $245 \pm 66 \mathrm{msec}$ ). For every cell except two (K6a and $\mathrm{K} 8$ ), located rostral of the middle SC, there were more spikes in the brake than in the control trials (Fig. 8, filled circles). The increase in number of spikes for most cells occurred, because plateaus provided an added discharge. For brake trials, in all of the cells, the number of spikes resulting when the plateaus were cut out resembled that in the control trials (Fig. 8, open circles). The reason rostral cells K6a and K8 did not show an increased spike count in brake trials can be easily appreciated from Figure $6 A 1$ showing the discharge of cell K6a. Because peak activity in control trials lagged gaze onset for this cell, there was a lowfrequency discharge at plateau onset and during the ensuing plateau that did not contribute significantly to the total spike count.

Behaviorally, the effect of the brake was to delay the time, relative to control trials, at which gaze acquired the target. The increased spike count was associated with a prolongation in the neural discharge of each cell, such that it took more time in brake trials to achieve the end of discharge. For example, for cell K2 (Figs. 2, 3), the overall discharge duration, measured from 10 msec before gaze onset to when activity decreased to $30 \%$ of its peak, was proportional to plateau duration (Fig. 9A). For the 12 caudal cells located $3 \mathrm{~mm}$ and more caudally in the SC (Table 1), the overall discharge duration, measured in this way, increased as 


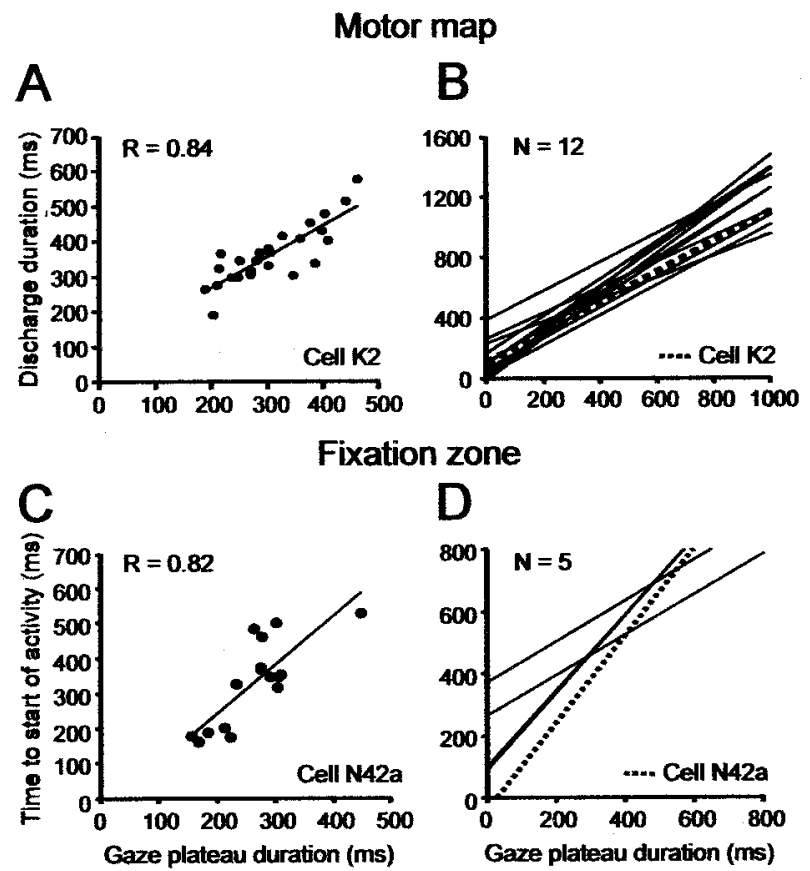

Figure 9. Effect of gaze plateau duration on cell discharges. Data from perturbed trials. $A$, Duration of overall cell discharge is proportional to gaze plateau duration (cell K2). Each point represents a single trial. Discharge duration, measured from $10 \mathrm{msec}$ before onset of first gaze saccade to when activity, during the postplateau corrective saccades, had decreased to $30 \%$ of peak discharge. Linear regression correlation coefficient $r=0.84$. $B$, Regression lines of discharge duration versus gaze plateau duration for all 12 cells in the caudal SC, posterior to the 3 mm location (Table 1). C, Dependence of the duration of fixation cell-suppressed activity (the pause) on gaze plateau duration (SCFN; cell N42a). Pause duration measured from gaze start to when activity had increased to $70 \%$ of its peak value. D, Same plot as in $A$ but for population of five SCFNs.

plateau duration increased (Fig. 9B). For the population of caudal cells, the linear regression equation with mean coefficients was as follows: duration discharge $=\left[(1.11 \pm 0.23) \times\right.$ duration $\left._{\text {plateau }}\right]+$ (123.8 \pm 114.1$)$; mean $r=0.81 \pm 0.14$. All of these neurons showed a significant linear regression correlation coefficient of $\geq 0.6$. We could not perform the simple duration analysis on cells in the middle SC, because there were two peaks in the discharge profile, and in between, the firing frequency could decline to near zero (Fig. $4 C$, bottom trace).

By extension with the prolonged discharge of cells on the motor map, the gaze shift-related silence of fixation cells increased during brake trials. For example, fixation cell N42a (Fig. 9C) showed an excellent linear relation between plateau duration and the time between onset of the gaze shift and when the activity of this neuron had resumed and built up to $70 \%$ of its peak value, the latter measured at gaze shift end. This relationship between gaze plateau duration and pause duration had a positive significant correlation $(p<0.05)$ in all five of our SCFNs (Fig. 9D). The mean regression line was as follows: duration pause $=[(1.0 \pm$ $0.35) \times$ duration $\left._{\text {plateau }}\right]+(158.8 \pm 160.1)$; mean $r=0.75 \pm 0.1$. [Recall that one of the two SCFNs in cat W was identified as a tectoreticular neuron (Table 1).]

Firing frequency varies with GPE

In the moving-hill hypothesis, the spatiotemporal pattern of firing frequency in the population of cells on the SC map encodes where gaze is relative to the ultimate goal of the gaze shift. This is implemented on the SC map by displacing, as a function of GPE, the locus of an ensemble of active tectoreticular neurons. For any
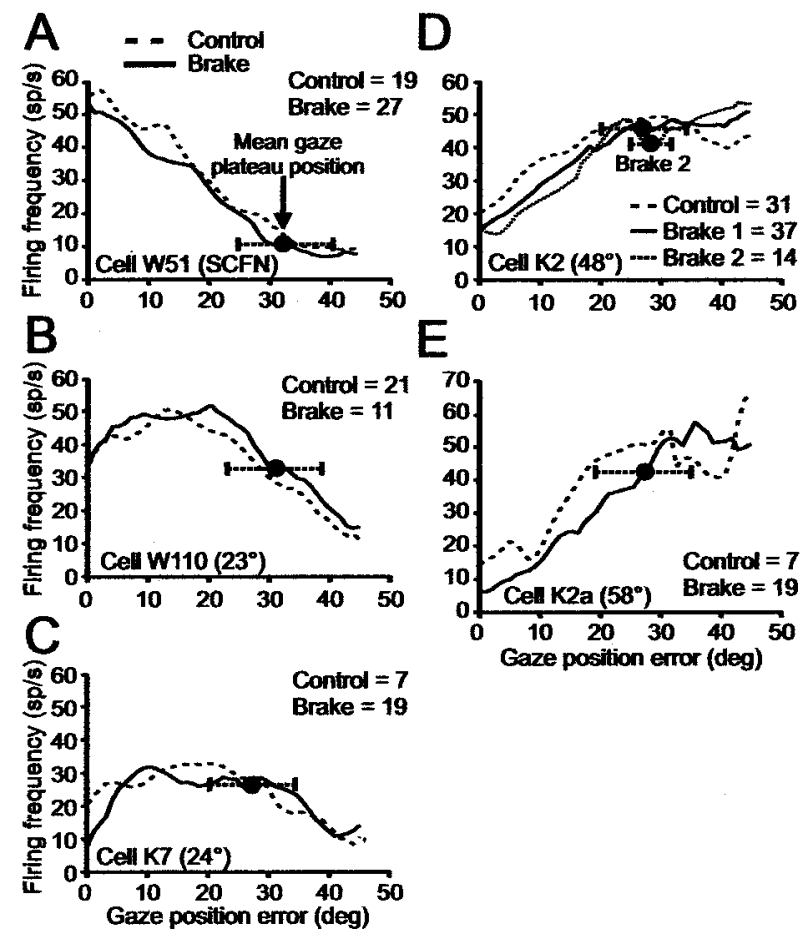

Figure 10. Phase plane plots showing dependence of firing frequency on GPE in control and brake trials. Time is not explicitly shown in these plots. Each panel shows data for one cell. $A-E$ are arranged in order of the rostrocaudal location of each cell on the SC map; fixation cell W51 is in $A$. In each panel, dashed and full lines show phase plane plots of average spike density for control and perturbed gaze shifts at each GPE. Number of trials in each condition is given in each panel. Mean plateau position is indicated by full circle with \pm 1 SD given by horizontal dotted line terminated by tic marks. In $D$, we selected a subset of 14 brake trials with plateau GPEs at $29 \pm 3^{\circ}$ (dotted line), a narrower range than for the solid line. Note for each panel that the phase plane plots in each condition are statistically identical (Materials and Methods) ( $p \geq$ $0.05 ; \mathrm{K}-\mathrm{S}$ test).

given cell, this mechanism results in the firing frequency during a gaze shift varying as a function of GPE, independent of whether the gaze shift is made as a single-step or as a multiple-step sequence. This phenomenon also holds true for the present data. Figure 10 shows mean phase plane plots (Materials and Methods) of firing frequency versus GPE for five cells ranked from top to bottom according to their rostrocaudal position on the map. The preferred GPEs of caudal cells K2 and K2a (Fig. 10D,E) were 48 and $58^{\circ}$, respectively, calculated from multiple-sep gaze shifts, but we could not obtain enough larger single-step control gaze shifts for these neurons to show that their firing frequency would drop off as GPE increased, thereby demonstrating a clear peak in this condition (Bergeron et al., 2003).

The brake trial plots (solid lines) in Figure 10 were obtained by cutting out both the plateaus in gaze trajectories and the corresponding time period in the firing-frequency histograms. This procedure assumes continuity in firing frequency between plateau start and end, which we justified previously in relation to our analysis of plateau firing frequency in Figure 7. The GPE of each plateau varied from trial to trial, and the mean \pm 1 SD is marked in each panel by the solid circle and the intersecting horizontal dotted line. For cell K2 (Fig. 10 D), we had enough brake trials to permit a comparison between the mean phase plane plots for all of the brake trials combined (solid line) with a subgroup of trials having a narrower distribution of plateau GPEs (dotted line and dark circle labeled Brake 2).

A K-S test (Materials and Methods) showed that the phase 
plane plots for the control and cut-out perturbed gaze shifts were identical ( $p \geq$ 0.05 ) for all of the SCFNs and for 17 of 20 cells on the motor map. No cell illustrated in Figure 10 had significant differences. That the control and brake trial plots are similar attests further to the continuity between the state of discharge at plateau start and end. This property is particularly striking for those cells in the middle SC (Fig. $9 B, C$ ) for which firing frequency during plateaus was not constant. The lower-than-control (but nonsignificant) firing frequency in the postplateau phase of caudal cells K2 and K2a of Figure 10 is typical of events in the caudal map.

The fact that the phase plane plots in control and brake trials were similar, once the plateaus in the latter have been cut out, needs to be interpreted in light of the observation that the gaze trajectories in the control and cut-out brake trials were also similar in the time domain (Fig. 6). Indeed, if the firing-frequency profile can vary with both time and GPE, then what is the relevant dependence? We will show in the next section that the moving-hill hypothesis unifies the different time- and GPE-dependent discharge properties of a cell.

Spatial distribution of activity on motor map during gaze shifts

We now show (Fig. 11) that the distribution of activity on the collicular map during a hypothetical $40^{\circ}$ single-step control gaze shift and a braked $40^{\circ}$ gaze shift supports the moving-hill hypothesis (Munoz et al., 1991a,b). For these calculations, we first determine the location of each cell on the collicular map on the basis of its preferred GPE (Materials and Methods). Given the controversy of the moving-hill hypothesis, we compared, for cells on the motor map, the outcome of using two different estimates of GPE: (1) in Figure $11 A-C$, from the GPE at peak firing frequency in the gaze position plateaus of multiplestep gaze shifts (Table 1, GPE Omsgs $_{\text {) }}$ (Table 1), and (2) in Figure $11 D-F$, from the mean amplitude of the gaze shift evoked by stimulating the recording site (Table $1, \mathrm{GPE}_{\mathrm{Ostim}}$ ).

Regarding SCFNs, note that the extent of the collicular fixation zone is unknown and might encompass a rostral zone 0.5$0.7 \mathrm{~mm}$ long in the rostrocaudal direction (Munoz and Wurtz, 1995b; Anderson et al., 1998). We assumed that SCFNs lie within a zone $0-0.5 \mathrm{~mm}$ long, and accordingly, we assigned to them random positions within this space along the horizontal meridian. Placing SCFNs at the zero location in the rostral pole did not significantly alter the conclusions of this section (data not shown).

To calculate the position of a cell using $\mathrm{GPE}_{\mathrm{Omsgs}}$ in the control condition (Fig. 11A-C), we had a total of 28 cells: 18 in Table 1 and 10 extra cells (Materials and Methods). For the same cal-

\section{Control}

Cell position
from GPE

Brake
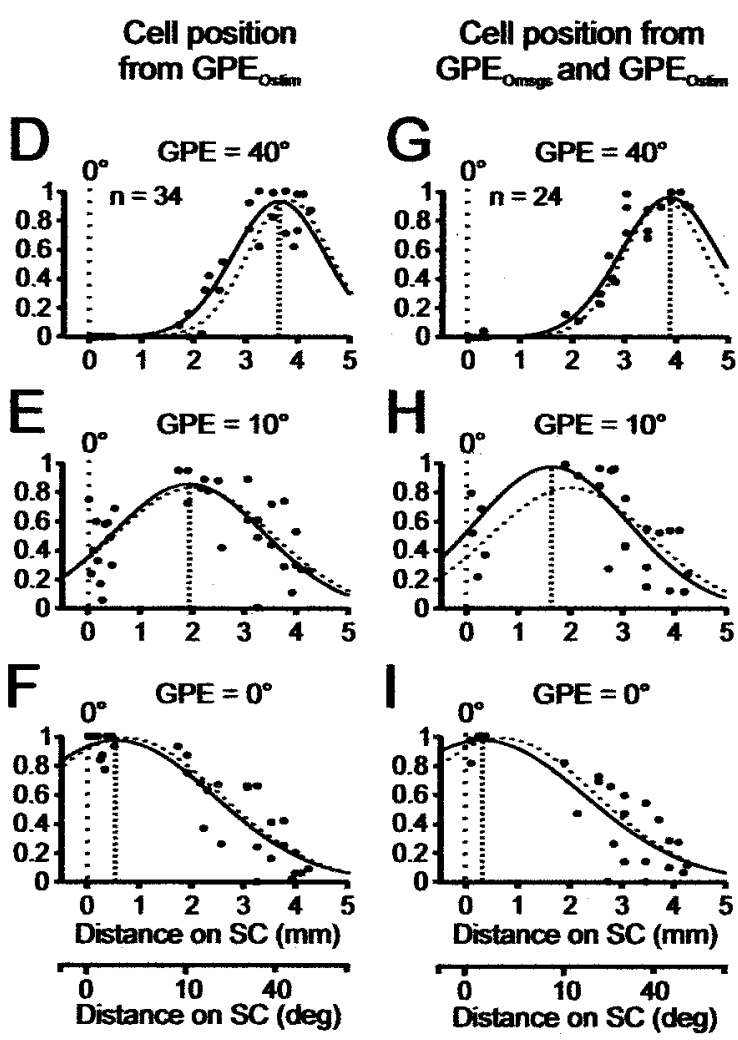

Figure 11. Spatial distribution of activity on the SC map at GPE of 40,10 , and $0^{\circ}$, respectively, during a $40^{\circ}$ gaze shift. The value normalized to its mean peak value obtained at its preferred GPE in either control (single-step gaze shift) trials ( $A-F$ ) or brake trials $-I)$. Abscissa, Location of each cell on the map determined by its preferred GPE determined from either its discharge charac distance in millimeters (Table 1) (Materials and Methods). Curve in each panel is the best-fit Gaussian through the points. $A-C$ Distribution of activity at different GPEs during a single-step $40^{\circ}$ control gaze shifts when cell location on map is determined from $\mathrm{GPE}_{0 \mathrm{msgs}}$. Number of available cells $n=28$; includes 18 cells in Table 1 and 10 extra cells (Materials and Methods). D-F, Same as in $A-C$, but with cell location determined by $\mathrm{GPE}_{0 \text { stim. }}$. Number of cells $n=34$; includes 24 from Table 1 and 10 extra cells. $G-I$,

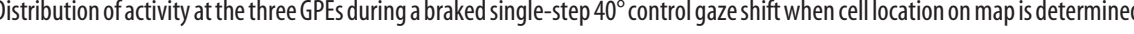

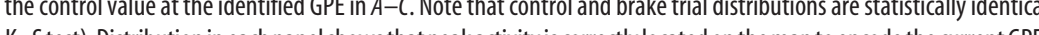
The peak moves caudorostrally along the map during gaze shifts, thereby keeping track of current GPE, and stops during brakeinduced plateaus. See Spatial Distribution of Activity on Motor Map during Gaze Shifts.

culation using $\mathrm{GPE}_{\mathrm{Ostim}}$, we had 34 cells: 24 in Table 1 and the 10 extra cells.

For each cell in Figure $11 A-F$, we obtained the firing frequency at a particular GPE from its control phase plane plot (Fig. 10) (Materials and Methods). The best-fit Gaussians to the data points in Figure 11, $A$ and $D$, show that, at the onset of a control $40^{\circ}$ gaze shift, activity was located at a site in the caudal SC appropriate for driving the entire movement. This is entirely consistent with classic views of SC function. At GPE of $10^{\circ}$ (Fig. $11 B, E)$, activity in both the rostral and $2-3 \mathrm{~mm}$ zones had increased, whereas that in about the $3.5-4.3 \mathrm{~mm}$ zone had decreased. As a result, peak activity was located in the middle SC. At gaze shift end $\left(\mathrm{GPE}=0^{\circ}\right)$ (Fig. $\left.11 C, F\right)$, activity in the rostral zone had increased further, and that in about the 2.5-4.3 zone had decreased even more, with the result that peak activity was approximately centered on the rostral fixation zone. The fact that, in the latter panels, the Gaussian was not quite centered at zero, when GPE $=0^{\circ}$, presumably was caused by noise in the best-fit 
procedure. The choice of GPE $=10^{\circ}$ was quite arbitrary, and we obtained similar results using different GPEs.

These calculations show that, in Figure 11, $A-C$ and $D-F$, the peak of activity moved progressively rostrally to different rostrocaudal locations for the three successive GPEs. The Gaussian curves were statistically indistinguishable in the two approaches to localizing the cells, as can be seen by visually comparing the solid and dotted profiles in $A$ and $D, B$ and $E$, and $C$ and $F$. Thus, the spatial distribution of firing frequency was indifferent to the method used to localize cells. It follows that activity during a control single-step gaze shift in cat moved continuously on the map in the caudorostral direction, from gaze shift start to finish, in agreement with previous observations (Munoz et al., 1991a,b).

Unfortunately, we lacked a sample of cells in the $0.5-2 \mathrm{~mm}$ zone, and our conclusions are based on the assumption of continuity between the properties of SCFNs and cells on the motor map caudal to $2.0 \mathrm{~mm}$. We believe it is unlikely that the discharge of cells in the 0.5-2 mm zone-if we had them-would have provided contradictory evidence, for two reasons: (1) in our initial proposal of the moving-hill phenomenon, our sample of cells covered the entire map, including this zone, and continuity of properties was observed (Munoz et al. 1991a,b); (2) during multiple-step gaze shifts, there is continuity between the discharge properties of SCFNs and cells on the motor map (Bergeron and Guitton 2000; Bergeron et al., 2003).

The distributions of activity on the map in Figure $11 G-I$ were calculated using data from brake trials only. (We included the 24 cells in Table 1 for which we had both control and brake data.) The mean values of GPE and their associated mean firing frequency for the brake trials were taken from data in which the plateaus had been cut out, as in Figure 10. Cells were localized using $\mathrm{GPE}_{\text {Omsgs }}$ when available (Table 1, 19 cells), but if this quantity was unavailable we used $\mathrm{GPE}_{\mathrm{Ostim}}$ ( 5 cells). Figure $11 G-I$ shows that activity during a brake trial behaved similarly to control trials and moved continuously rostrally on the map beginning at a caudal starting position encoding overall gaze shift amplitude. Given that activity at plateau end equaled that at plateau start (Fig. 10), we conclude that the caudorostral movement stopped during a plateau and resumed moving rostrally at the end of the plateau. The gaze shift terminated with activity located in the rostral pole. In the period between brake onset and plateau end, activity in primarily the middle region of the map underwent a transient modulation not shown in Figure $11 G-I$.

Note that the moving-hill hypothesis unifies the different time- and GPE-dependent discharge properties of a cell (Figs. 6, 10). The position on the map of the peak of the hill encoded the current GPE, independent of the temporal profile of a trajectory, and variability in the kinematics of the profile was mirrored in the kinematic properties of the translational motion of the locus of activity on the map.

\section{Discussion}

\section{Compensation for gaze perturbations: the SC and the gaze} feedback hypothesis

There is now considerable evidence supporting the hypothesis that saccadic gaze shifts made with coordinated eye-head movements are driven by an error signal (GPE) derived from a gaze feedback loop that compares the current gaze vector with the desired vector (for review, see Scudder et al., 2002; Guitton et al., $2003 a, b)$. One test of this gaze feedback hypothesis is to perturb, unexpectedly to the subject, gaze trajectories made in the dark and verify that the system compensates for the perturbations such that gaze accuracy remains unaltered relative to control. Such experiments have been done in cat, monkey, and humans (for review, see Guitton et al., 2003a,b). The accurate corrective gaze saccades in the present head brake experiments provide not only additional behavioral support for the gaze feedback control concept but also assure that the neuronal recordings made during the head perturbations were obtained in the context of an operational feedback system.

Keller (1981) first proposed that the SC in the head-fixed primate is within an eye saccade feedback loop. This concept has been tested in the head-fixed monkey by perturbing saccade trajectories and examining whether cell discharges respond to perturbations and correlate to compensatory movements. The results are controversial. For example, eye blinks result in concurrent changes in both saccade trajectories and collicular saccade-related discharges (Goossens and Van Opstal, 2000a,b). Saccades do compensate for blink-induced perturbations, but such observations have been explained by suggesting that blinkrelated signals themselves modify firing-frequency patterns of SC cells, and that the internal circuitry of the SC emits a site-specific constant number of spikes without receiving feedback from the saccade burst generator. Note that the present observation showing an increased number of spikes in brake trials (Fig. 8) opposes this assumption for perturbed gaze shifts.

In another approach for investigating feedback to the primate SC, saccades have been interrupted by electrically stimulating either the omnipause neuron region or the fixation zone of the SC (Keller and Edelman, 1994; Munoz et al., 1996; Keller et al., 2000) (for review, see Scudder et al., 2002). After the cessation of stimulation, accurate corrective saccades were generated. The discharge of collicular burst neurons was interrupted by stimulation. Activity resumed at the same SC site just before and synchronously with the corrective saccade. In comparison, $\sim 30 \%$ of the collicular buildup neurons were not suppressed by stimulation, and their activity continued tonically throughout the interruption period. These results have been used to support the hypothesis of feedback to the primate SC (Munoz et al., 1996; Keller et al., 2000) but were unclear regarding what feedback encodes.

In comparison, Soetedjo et al. (2002a) slowed saccades by deactivating the primate omnipause neuron area using pharmacological techniques. The reason saccades were slowed is unknown. The duration of cell discharges was correspondingly prolonged, showing that the SC received information about the slowed trajectory. The authors argued that feedback to the SC encodes saccade end, not motor error.

In summary, the results of different experiments that perturbed saccade trajectories have been interpreted differently, and the debate continues as to whether the primate SC participates in the on-line feedback control of eye saccades. One reason for the debate is that the technique of perturbing saccade trajectories in the head-fixed primate is subject to artifacts. For example, in the electrical stimulation experiments, neurons that project to the stimulated site and that themselves are involved in driving the movement can be directly affected by stimulation (e.g., antidromically). Such problems are avoided when head-free gaze shifts are perturbed by obstructing head motion, a common reallife phenomenon experienced in many sporting activities that require precise visuomotor control.

Using head brakes, we provided in this paper the following evidence for gaze feedback to the cat SC. First, for virtually all of the cells on the map, the discharge pattern in perturbed movements was very different from that in controls (Fig. 6) (i.e., the SC was informed about the perturbation). Second, the pause in 
SCFNs was prolonged in proportion to plateau duration (Figs. 5, $9 C, D)$. Third, the discharge of cells on the motor map was prolonged relative to control (Figs. 6, 9A,B), and the total number of spikes emitted by a cell was greater than control and increased with plateau duration (Fig. 8). In comparison, when the number of spikes in the plateaus was subtracted out, the total number of spikes emitted in the perturbed and control conditions became about equal (Fig. 8).

Recall that the cells we describe here had discharge properties similar to those of cat tectoreticular neurons (Grantyn and Berthoz, 1985; Munoz et al., 1991b; Olivier et al., 1993), and we verified this in cat $\mathrm{W}$ in which we antidromically identified some cells as tectoreticular neurons. Therefore, feedback occurred on collicular output cells.

\section{Cat SC encodes GPE because of feedback}

Although the brake experiments demonstrated feedback to the cat SC, the question arises as to whether this feedback implemented the encoding of GPE via the moving-hill phenomenon. In this model, derived in the head-unrestrained cat, the locus of the population activity is continuously adjusted by feedback to be centered on the appropriate instantaneous GPE [modeled by Lefèvre and Galiana (1992)]. The gaze shift ends whenever $\mathrm{GPE}=0^{\circ}$, and the locus of moving neural activity has invaded the zero of the map, the rostral pole of the SC where SCFNs are located.

The present control discharge patterns are compatible with this model. Indeed, an analysis of the spatial distribution of the population discharge on the map showed that the locus of the peak firing frequency progressed rostrally as GPE decreased (Fig. 11). This result is compatible with that of Munoz et al. (1991a,b), who studied single-step gaze shift in cat, and with Bergeron and Guitton (2000) and Bergeron et al. (2003), who studied multiplestep gaze shifts. As a result of the moving bell-shaped hill, the firing frequency of all of the cells (including SCFNs) presented a stereotyped, position-dependent relationship between firing frequency and GPE (Fig. 10). Furthermore, the firing frequency of SCFNs gradually increased as GPE approached $0^{\circ}$ and peaked at gaze end. Finally, for large $\left(\sim 50^{\circ}\right)$ control gaze shifts, the peak firing frequency of cells in the middle SC was delayed (Figs. 4, 6) relative to gaze onset.

For the brake trials, the pattern of activity before and after the brake was applied was the same as control. The phase plane plots of firing frequency versus GPE were statistically indistinguishable from control data in 22 of 24 cells. Thus, in most cells, the brake and its arrest of gaze motion did not significantly modify the postplateau neural activity relative to control gaze shifts at the same GPE. In spite of the fact that, for some cells, the activity during plateaus was not constant, the population activity at the very end of a plateau, for both middle and caudal cells, was equal to that at the start of the plateau (Fig. 7), that is, activity at plateau end, just when gaze got underway again at the start of the corrective gaze saccade, was at control values determined by GPE. The results imply that, in brake trials, the rostrally moving hill must have stopped during plateaus. Our analysis showed further that the progression of activity on the map was similar in multiplestep gaze shifts (Bergeron et al., 2003) and in the present brake trials, implying no difference in the encoding of GPE in naturally and artificially interrupted gaze shifts.

\section{The primate SC and the encoding of GPE}

By analogy with the moving hill in cat initially described by $\mathrm{Mu}-$ noz et al. (1991a,b), Munoz and Wurtz (1995b) described for monkey a moving front of activity that spread across the buildup neuron layer toward the rostral pole of the SC as the saccade progressed. In comparison, activity in the collicular burst neuron layer remained topographically immobile during a saccade. Rostrally moving activity during saccades in monkey has been found in experiments involving the simultaneous recording of activity in two buildup neurons (Port et al., 2000), but what this phenomenon encoded was not clear. No other investigation has succeeded in showing significant rostral movement of the locus of activity during eye saccades [imaging SC (Moschovakis et al., 2001); single-cell recording (Anderson et al., 1998; Soetedjo et al., 2002b)], even less the encoding of GPE. Furthermore, deactivating the rostral SC did not lead to hypermetric saccades as predicted by the hypothesis (Munoz and Wurtz, 1993; Aizawa and Wurtz, 1998; Quaia et al., 1998). Therefore, for the head-fixed monkey, the moving-hill model has little support, although there is evidence for feedback to the SC (Keller and Edelman, 1994; Munoz et al., 1996; Keller et al., 2000; Soetedjo et al., 2002a), which the latter authors have suggested controls saccade termination. By extension, in the head-unrestrained monkey, we provided preliminary evidence, using the same experimental technique as here (Choi and Guitton, 2002; Guitton and Choi, 2002; Guitton et al., 2003a,b), that there is gaze feedback to the SC during brake-induced gaze plateaus. However, evidence for a moving locus of activity that encodes GPE in the headunrestrained monkey, if it exists, is far less striking than in cat. Understanding the basis of such species differences could provide original insights into collicular function.

\section{References}

Aizawa H, Wurtz RH (1998) Reversible inactivation of monkey superior colliculus. I. Curvature of saccadic trajectory. J Neurophysiol 79:2082-2096.

Anderson RW, Keller EL, Gandhi NJ, Das S (1998) Two-dimensional saccade-related population activity in superior colliculus in monkey. J Neurophysiol 80:798-817.

Bergeron A, Guitton D (2000) Fixation neurons in the superior colliculus encode distance between current and desired gaze positions. Nat Neurosci 3:932-939.

Bergeron A, Guitton D (2002) In multiple-step gaze shifts: omnipause (OPNs) and collicular fixation neurons encode gaze position error; OPNs gate saccades. J Neurophysiol 88:1726-1742.

Bergeron A, Matsuo S, Guitton D (2003) Superior colliculus encodes distance to target, not saccade amplitude, in multi-step gaze shifts. Nat Neurosci 6:404-413.

Berman AL (1968) The brainstem of the cat. Madison, WI: University of Wisconsin.

Choi WY, Guitton D (2002) Discharge characteristics of saccade-related neurons in primate superior colliculus during head-perturbed gaze shifts. Soc Neurosci Abstr 28:11.7.

Cullen KE, Guitton D (1996) Inhibitory burst neuron activity encodes gaze, not eye, metrics and dynamics during passive head on body rotation. Evidence that vestibular signals supplement visual information in the control of gaze shifts. Ann NY Acad Sci 781:601-606.

Feldon S, Feldon P, Kruger L (1970) Topography of the retinal projection upon the superior colliculus of the cat. Vision Res 10:135-143.

Goossens HH, Van Opstal AJ (2000a) Blink-perturbed saccades in monkey. I. Behavioral analysis. J Neurophysiol 83:3411-3429.

Goossens HH, Van Opstal AJ (2000b) Blink-perturbed saccades in monkey. II. Superior colliculus activity. J Neurophysiol 83:3430-3452.

Grantyn A, Berthoz A (1985) Burst activity of identified tecto-reticulospinal neurons in the alert cat. Exp Brain Res 57:417-421.

Guitton D (1991) Control of saccadic eye and gaze movements by the superior colliculus, basal ganglia. In: Vision and visual dysfunction. Eye movements (Carpenter RHS, ed), pp 244-276. London: MacMillan.

Guitton D (1992) Control of eye-head coordination during orienting gaze shifts. Trends Neurosci 15:174-179.

Guitton D, Choi WY (2002) Discharge characteristics of fixation neurons in 
primate superior colliculus during head-perturbed gaze shifts. Soc Neurosci Abstr 28:11.8.

Guitton D, Douglas RM, Volle M (1984) Eye-head coordination in cats. J Neurophysiol 52:1030-1050.

Guitton D, Bergeron A, Choi WY, Matsuo S (2003a) On the feedback control of orienting gaze shifts made with eye and head movements. Prog Brain Res 142:55-68.

Guitton D, Bergeron A, Choi WY (2003b) On the role of subcortical feedback mechanisms in the control of gaze saccades made headunrestrained. In: The superior colliculus: new approaches for studying sensorimotor integration (Hall WC, ed), pp 241-276. Boca Raton, FL: CRC.

Keller EL (1981) Brain stem mechanisms in saccadic control. In: Progress in oculomotor research, developments in neuroscience (Fuchs AF, Becker W, eds), pp 57-62. New York: Elsevier/North-Holland Biomedical.

Keller EL, Edelman JA (1994) Use of interrupted saccade paradigm to study spatial and temporal dynamics of saccadic burst cells in superior colliculus in monkey. J Neurophysiol 72:2754-2770.

Keller EL, Gandhi NJ, Vijay Sekaran S (2000) Activity in deep intermediate layer collicular neurons during interrupted saccades. Exp Brain Res 130:227-237.

Lefèvre P, Galiana HL (1992) Dynamic feedback to the superior colliculus in a neural network model of the gaze control system. Neural Netw 5:871-890.

Lipski J (1981) Antidromic activation of neurones as an analytic tool in the study of the central nervous system. J Neurosci Methods 4:1-32.

MacPherson JM, Aldridge JW (1979) A quantitative method of computer analysis of spike train data collected from behaving animals. Brain Res 175:183-187.

Moschovakis AK, Scudder CA, Highstein SM (1996) The microscopic anatomy and physiology of the mammalian saccadic system. Prog Neurobiol 50:133-254

Moschovakis AK, Gregoriou GG, Savaki HE (2001) Functional imaging of the primate superior colliculus during saccades to visual targets. Nat Neurosci 4:1026-1031.

Munoz DP, Guitton D (1991) Control of orienting gaze shifts by the tectoreticulospinal system in the head-free cat. II. Sustained discharges during motor preparation and fixation. J Neurophysiol 66:1624-1641.

Munoz DP, Wurtz RH (1993) Fixation cells in monkey superior colliculus. II. Reversible activation and deactivation. J Neurophysiol 70:576-589.

Munoz DP, Wurtz RH (1995a) Saccade-related activity in monkey superior colliculus. I. Characteristics of burst and buildup cells. J Neurophysiol 73:2313-2333.

Munoz DP, Wurtz RH (1995b) Saccade-related activity in monkey superior colliculus. II. Spread of activity during saccades. J Neurophysiol 73:2334-2348

Munoz DP, Pélisson D, Guitton D (1991a) Movement of neural activity on the superior colliculus motor map during gaze shifts. Science 251:1358-1360.

Munoz DP, Guitton D, Pélisson D (1991b) Control of orienting gaze shifts by the tectoreticulospinal system in the head-free cat. III. Spatiotemporal characteristics of phasic motor discharges. J Neurophysiol 66:1642-1666.

Munoz DP, Waitzman DM, Wurtz RH (1996) Activity of neurons in monkey superior colliculus during interrupted saccades. J Neurophysiol 75:2562-2580.

Olivier E, Grantyn A, Chat M, Berthoz A (1993) The control of slow orienting eye movements by tectoreticulospinal neurons in the cat: behavior, discharge patterns and underlying connections. Exp Brain Res 93:435-449.

Paré M, Crommelinck M, Guitton D (1994) Gaze shifts evoked by stimulation of the superior colliculus in the head-free cat conform to the motor map but also depend on stimulus strength and fixation activity. Exp Brain Res 101:123-139.

Peck CK (1987) Saccade related burst neurons in cat superior colliculus. Brain Res 408:329-333.

Port NL, Sommer MA, Wurtz RH (2000) Multielectrode evidence for spreading activity across the superior colliculus movement map. J Neurophysiol 84:344-357.

Quaia C, Aizawa H, Optican LM, Wurtz RH (1998) Reversible inactivation of monkey superior colliculus. II. Maps of saccadic deficits. J Neurophysiol 79:2097-2110.

Richmond BJ, Optican LM, Podell M, Spitzer H (1987) Temporal encoding of two dimensional patterns by single units in primate inferior temporal cortex. I. Response characteristics. J Neurophysiol 57:132-146.

Robinson DA (1963) A method of measuring eye movements using a scleral search coil in a magnetic field. IEEE Trans Biomed Eng 10:137-145.

Scudder CA, Kaneko CS, Fuchs AF (2002) The brainstem burst generator for saccadic eye movements. A modern synthesis. Exp Brain Res 142:439-462.

Soetedjo R, Kaneko CR, Fuchs AF (2002a) Evidence that the superior colliculus participates in the feedback control of saccadic eye movements. J Neurophysiol 87:679-695.

Soetedjo R, Kaneko CR, Fuchs AF (2002b) Evidence against a moving hill in the superior colliculus during saccadic eye movements in the monkey. J Neurophysiol 87:2778-2789.

Waitzman DM, Ma TP, Optican LM, Wurtz RH (1991) Superior colliculus neurons mediate the dynamic characteristics of saccades. J Neurophysiol 66:1716-1737. 